\title{
CSI 2264: CHARACTERIZING YOUNG STARS IN NGC 2264 WITH STOCHASTICALLY VARYING LIGHT CURVES*
}

\author{
John Stauffer ${ }^{1}$, Ann Marie Cody $^{2}$, Luisa Rebull ${ }^{1}$, Lynne A. Hillendrand ${ }^{3}$, Neal J. Turner ${ }^{4}$, John Carpenter ${ }^{3}$, \\ Sean Carey $^{1}$, Susan Terebey ${ }^{5}$, María Morales-Calderón ${ }^{6}$, Silvia H. P. Alencar ${ }^{7}$, Pauline McGinnis ${ }^{7}$, Alana Sousa ${ }^{7}$, \\ Jerome Bouvier ${ }^{8,9}$, Laura Venuti ${ }^{8,9}$, Lee Hartmann $^{10}$, Nuria Calvet $^{10}$, Giusi Micela ${ }^{11}$, Ettore Flaccomio ${ }^{11}$, \\ Inseok Song ${ }^{12}$, Rob Gutermuth ${ }^{13}$, David Barrado ${ }^{6}$, Frederick J. Vrba ${ }^{14}$, KeVin Covey ${ }^{15}$, William Herbst ${ }^{16}$, \\ Edward Gillen ${ }^{17}$, Marcelo Medeiros Guimarães ${ }^{18}$, Herve Bouy $^{6}$, and Fabio Favata ${ }^{19}$ \\ ${ }^{1}$ Spitzer Science Center, California Institute of Technology, Pasadena, CA 91125, USA; stauffer@ipac.caltech.edu \\ ${ }^{2}$ NASA Ames Research Center, Kepler Science Office, Mountain View, CA 94035, USA \\ ${ }^{3}$ Astronomy Department, California Institute of Technology, Pasadena, CA 91125, USA \\ ${ }^{4}$ Jet Propulsion Laboratory, California Institute of Technology, Pasadena, CA 91109, USA \\ ${ }^{5}$ Department of Physics and Astronomy, 5151 State University Drive, California State University at Los Angeles, Los Angeles, CA 90032, USA \\ ${ }^{6}$ Centro de Astrobiología, Dpto. de Astrofísica, INTA-CSIC, P.O. BOX 78, E-28691, ESAC Campus, Villanueva de la Cañada, Madrid, Spain \\ ${ }^{7}$ Departamento de Física-ICEx-UFMG, Av. Antônio Carlos, 6627, 30270-901, Belo Horizonte, MG, Brazil \\ ${ }^{8}$ Université de Grenoble, Institut de Planétologie et d'Astrophysique de Grenoble (IPAG), F-38000 Grenoble, France \\ ${ }^{9}$ CNRS, IPAG, F-38000 Grenoble, France \\ ${ }^{10}$ Department of Astronomy, University of Michigan, 500 Church Street, Ann Arbor, MI:48105, USA \\ ${ }_{11}^{11}$ INAF-Osservatorio Astronomico di Palermo, Piazza del Parlamento 1, I-90134, Palermo, Italy \\ ${ }^{12}$ Department of Physics and Astronomy, The University of Georgia, Athens, GA 30602-2451, USA \\ ${ }^{13}$ Department of Astronomy, University of Massachusetts, Amherst, MA 01003, USA \\ ${ }^{14}$ U.S. Naval Observatory, Flagstaff Station, 10391 West Naval Observatory Road, Flagstaff, AZ 86001, USA \\ ${ }^{15}$ Department of Physics and Astronomy (MS-9164), Western Washington Univ., 516 High St., Bellingham, WA 98225, USA \\ ${ }^{16}$ Astronomy Department, Wesleyan University, Middletown, CT 06459, USA \\ ${ }^{17}$ Department of Physics, University of Oxford, Keble Road, Oxford, OX1 3RH, UK \\ ${ }^{18}$ Departamento de Física-UFS-Rod. Marechal Rondon, 49100-000, São Cristovão, SE, Brazil \\ ${ }^{19}$ European Space Agency, 8-10 rue Mario Nikis, F-75738 Paris Cedex 15, France \\ Received 2015 October 12; accepted 2016 January 7; published 2016 February 18
}

\begin{abstract}
We provide CoRoT and Spitzer light curves and other supporting data for 17 classical T Tauri stars in NGC 2264 whose CoRoT light curves exemplify the "stochastic" light curve class as defined in 2014 by Cody et al. The most probable physical mechanism to explain the optical variability within this light curve class is time-dependent mass accretion onto the stellar photosphere, producing transient hot spots. Where we have appropriate spectral data, we show that the veiling variability in these stars is consistent in both amplitude and timescale with the optical light curve morphology. The veiling variability is also well-correlated with the strength of the He I $6678 \AA$ emission line, predicted by models to arise in accretion shocks on or near the stellar photosphere. Stars with accretion burst light curve morphology also have variable mass accretion. The stochastic and accretion burst light curves can both be explained by a simple model of randomly occurring flux bursts, with the stochastic light curve class having a higher frequency of lower amplitude events. Members of the stochastic light curve class have only moderate mass accretion rates. Their $\mathrm{H} \alpha$ profiles usually have blueshifted absorption features, probably originating in a disk wind. The lack of periodic signatures in the light curves suggests that little of the variability is due to long-lived hot spots rotating into or out of our line of sight; instead, the primary driver of the observed photometric variability is likely to be instabilities in the inner disk that lead to variable mass accretion.
\end{abstract}

Key words: circumstellar matter - open clusters and associations: individual (NGC 2264) - stars: pre-main sequence - stars: protostars - stars: variables: T Tauri, Herbig Ae/Be

\section{INTRODUCTION}

The published literature on the photometric variability of $\mathrm{T}$ Tauri stars now extends back in time more than 150 years (Schmidt 1861, 1866), predating by nearly 100 years the general acceptance that $\mathrm{T}$ associations are sites of recent/current star formation (Ambartsumian 1954; Salpeter 1954), and therefore that

\footnotetext{
* Based on data from the Spitzer and CoRoT missions, as well as the CanadaFrance-Hawaii Telescope (CFHT) MegaCam CCD, and the European Southern Observatory Very Large Telescope, Paranal Chile, under program 088.C-0239. The CoRoT space mission was developed and is operated by the French space agency CNES, with particpiation of ESA's RSSD and Science Programmes, Austria, Belgium, Brazil, Germany, and Spain. MegaCam is a joint project of CFHT and CEA/DAPNIA, which is operated by the National Research Council (NRC) of Canada, the Institute National des Sciences de l'Univers of the Centre National de la Recherche Scientifique of France, and the University of Hawaii.
}

the photometric variability of $\mathrm{T}$ Tauri stars is likely a signpost of youth (Walker 1956). Haro \& Herbig (1955) and Walker (1956) discovered that many of the photometrically variable $\mathrm{T}$ Tauri stars also had UV excesses and abnormally weak photospheric absorption lines (now usually described as spectral "veiling"); the former authors concluded that the UV excesses might originate from a small hot spot on or near the stellar photosphere. However, it was not until the 1970s that the modern paradigm for young stellar objects (YSOs) began to develop, first by linking the photospheric hotspots to accretion from a disk (Walker 1972; Wolf et al. 1977), and eventually by having the accretion stream be channeled by magnetic field lines connecting the star and inner disk (Ghosh \& Lamb 1978; Königl 1991).

Based on photometry derived from visual observations or photographic plates, Joy (1945) made photometric variability 
one of the defining characteristics of the T Tauri class of stars. Only the largest amplitude variables could be identified with those techniques. The modern era in the study of the photometric variability of T Tauri stars began in the $1980 \mathrm{~s}$ in a series of papers by Herbst and his students (Herbst et al. 1982, 1987; Holtzman et al. 1986) and by Vrba and his collaborators (Rydgren \& Vrba 1983; Vrba et al. 1986, 1989). They showed that very young stars often have spotted photospheres, allowing their rotation periods to be derived (which showed that many of them are rapidly rotating relative to the Sun, as had long been known from spectroscopy, e.g., Walker 1956). In many cases, the data were compatible with cold spots as found on the Sun; however, in some cases-particularly when photometry over a broad wavelength range was available - the data were best fit by spots hotter than the stellar photosphere, confirming the speculation of Haro \& Herbig (1955). Other T Tauri stars instead showed apparently chaotic (or at least aperiodic) variability. Herbst et al. (1994) summarized the existing data on $\mathrm{T}$ Tauri variability, and proposed sorting the light curves into several physically motivated classes:

1. Type I-light curves resulting from stable, long-lived cold spots that are not distributed in an axisymmetric way over the stellar photosphere. The resultant light curves are periodic, and persist with little change often for months to years;

2. Type II-light curves dominated by relatively short lived hot spots. Often these do not show obvious periodicity, indicating that the hot spots evolve significantly on timescales less than the stellar rotation period;

3. Type IIp-hot spot light curves where the spot lifetime is instead comparable to or larger than the stellar rotation period, resulting in more or less periodic variations-but which generally evolve rapidly;

4. Type III-young stars with large amplitude flux variations in their light curves which are not accompanied by significant variations in the veiling inferred from spectroscopy. The physical interpretation of this class was uncertain, though the leading candidate was extinction by clumps of dust (most plausibly connected to structures in the inner circumstellar disk).

In the past two decades, time series photometry in starforming regions has been used primarily to determine rotation periods in order to study the initial angular momentum distribution for low mass stars (Herbst et al. 2000; Rebull 2001; Lamm et al. 2005; Moraux et al. 2013). Those programs have been quite successful, with rotation periods now known for more than a thousand low mass stars with ages less than a few Myr. Some programs have continued to attempt to characterize YSO variability not due to spots (e.g., Carpenter et al. 2001), including a series of campaigns aimed at a particularly intriguing YSO_AA Tau (Bouvier et al. 1999; Terquem \& Papaloizou 2000; Bouvier et al. 2003). Based on intensive ground-based time series photometry and spectroscopy over several years, the latter set of papers provided evidence that the light curve for AA Tau is dominated by variable extinction caused by a warped inner disk periodically intersecting our line of sight. More recently, using three weeks of continuous, high signal-to-noise ratio $(\mathrm{S} / \mathrm{N})$ photometry from the CoRoT satellite, Alencar et al. (2010) showed that many of the classical T Tauri stars (CTTS) in the star-forming region NGC 2264 have light curves similar to AA Tau, indicating that variable extinction from structures in the circumstellar disk passing through our line of sight is common.

This paper is based on a number of observational efforts organized around a Spitzer Space Telescope Exploration Science program entitled "A Coordinated, Synoptic Investigation of Star Formation in NGC 2264" (aka CSI 2264). CSI 2264 combines simultaneous or contemporaneous time series observations of the entire NGC 2264 star-forming region spanning a wide range in wavelength and including both photometry and spectroscopy. Cody et al. (2014) provide an overview of the program and propose a new, quantitative light curve classification scheme for YSOs based on indices that measure the degree of periodicity and the degree of symmetry of the light curves. Stauffer et al. (2014) identified and characterized the members of one of these classes-CTTS whose light curves are dominated by short-duration flux bursts. McGinnis et al. (2015) and Stauffer et al. (2015) similarly identify and characterize CTTS whose light curves are dominated by variable extinction events. L. Venuti et al. (2016, in preparation) discuss all of the NGC 2264 stars whose CoRoT light curves are periodic or quasi-periodic. Figure 1 illustrates stars whose light curves are dominated by variable extinction, accretion bursts, cold or hot spots, and a prototype of the stochastic light curve class, the topic of this paper.

The last remaining, major light curve class identified by Cody et al. (2014) for which our group has not yet published a detailed characterization paper are the stars designated as having stochastic light curves. Light curves in this class show variability with no definite periodicity and also show symmetric shapes (that is, they do not appear to have a continuum level punctuated by dips-as for the variable extinction stars-nor a continuum level punctuated by discrete flux excesses - as for the accretion burst stars). The goal of this paper is to illustrate in more detail the characteristics of the stars with stochastic optical light curves, and to propose a physical mechanism for their variability. In Section 2, we give a brief listing of the observational data obtained as part of the CSI 2264 program. In Section 3, we identify a set of seventeen CTTS whose light curves we consider to exemplify the stochastic class, we provide additional empirical data for these stars, and we compare their properties to other CTTS members of NGC 2264. We believe the most plausible physical mechanism to explain the optical variability of the stars with stochastic light curves to be variable accretion onto the star's photosphere, and we provide our arguments for that conclusion in Section 4. Additional characterization of the stochastic light curve stars is provided in Section 5. In Section 6, we summarize the properties of the stochastic light curve class members and discuss connections to physical models and how future observations could improve our understanding of these stars.

\section{OBSERVATIONAL DATA}

A detailed description of the data we collected in our 2008 and 2011 observing campaigns for the NGC 2264 star-forming region is provided in Cody et al. (2014). ${ }^{20}$ The subset of the data sources that we use here is based primarily on the synoptic

\footnotetext{
20 The CoRoT and Spitzer light curves for all probable NGC 2264 members, as well as our broad band photometry for these stars, are available at http://irsa. ipac.caltech.edu/data/SPITZER/CSI2264. As of 2015 June, calibrated versions of all VLT/FLAMES spectra obtained prior to 2014 Decemberincluding those taken for both CSI 2264 and ESO-Gaia-are available at http://archive.eso.org/wdb/wdb/adp/phase3_spectral.
} 

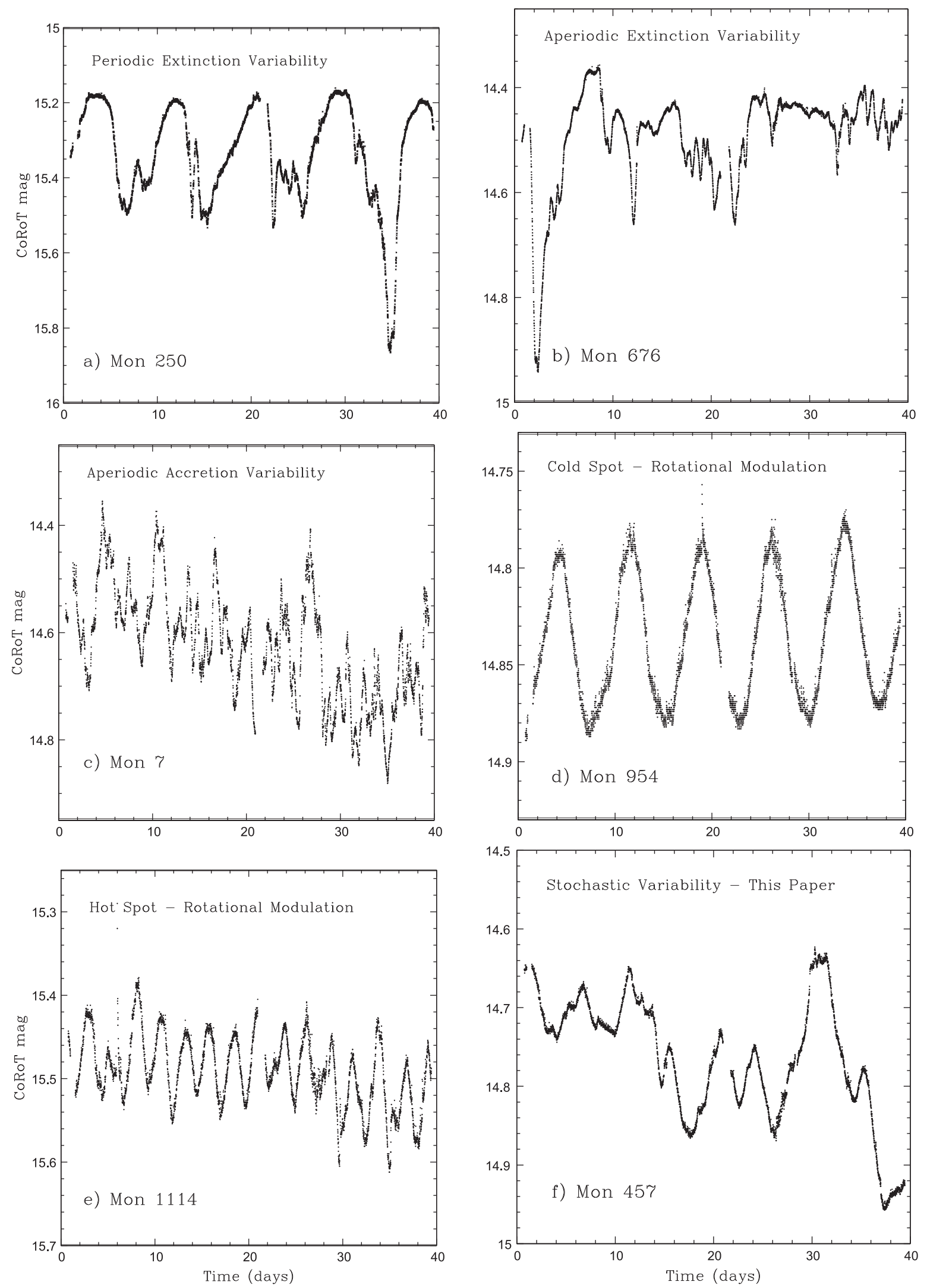

Figure 1. Light curves for five CTTS members of NGC 2264 whose CoRoT light curves illustrate morphologies to which we have ascribed physical mechanisms including variable extinction (panels (a) and (b)), unstable accretion or accretion burst (panel (c)), cold spots (panel (d)), and hot spots (panel (e)), and a sixth CTTS that shows a typical stochastic light curve (panel (f)). The variable extinction exemplars include an AA Tau analog (Mon-250) and a star with aperiodic flux dips possibly due to instability events briefly raising dust structures above the quiescent disk photosphere (Mon-676). The Mon-1114 light curve also includes two isolated short-duration flux dips near days 30 and 35 of the type which we associate with extinction due to dust entrained in accretion streams (Stauffer et al. 2015). 
Table 1

Synoptic Data Used in This Paper

\begin{tabular}{lccccc}
\hline \hline Telescope & Data Type & Wavelength(s) & Resolution & $\begin{array}{c}\text { Date Range } \\
\text { (MJD) }\end{array}$ \\
\hline CoRoT & Optical Photometry & $6000 \AA$ & 2 & $54534-54556$ & Cadence \\
CoRoT & Optical Photometry & $6000 \AA$ & 2 & $55897-55936$ & $32 \mathrm{~s}$ or $512 \mathrm{~s}$ \\
Spitzer & IR Photometry & 3.6 and $4.5 \mu \mathrm{m}$ & 4 & $55900-55930$ & $32 \mathrm{~s}$ or $512 \mathrm{~s}$ \\
USNO $1.0 \mathrm{~m}$ & Optical Photometry & $7000 \AA$ & 8 & $55890-55990$ & $2 \mathrm{hr}$ \\
CFHT & Optical Photometry & ugri & 10 & $55971-55986$ & few dozen per night \\
VLT/FLAMES & Spectroscopy & $6450-6750 \AA$ & 17000 & $55899-55981$ & $\sim 20$ times per night \\
VLT/FLAMES & Spectroscopy & $6450-6750 \AA$ & 17000 & $56648-56660$ & $\sim 1$ spectrum per night \\
\hline
\end{tabular}

Table 2

Basic Information for YSOs with Stochastic Optical Light Curve Shapes

\begin{tabular}{|c|c|c|c|c|c|c|c|c|c|}
\hline Mon $\mathrm{ID}^{\mathrm{a}}$ & 2MASS ID & CoRoT $^{\mathrm{b}}$ & $\mathrm{SpT}^{\mathrm{c}}$ & $\begin{array}{c}\mathrm{H} \alpha \mathrm{EW}^{\mathrm{c}} \\
(\AA)\end{array}$ & FR $4.5^{\mathrm{d}}$ & $\begin{array}{l}\text { CoRoT Ampl. } \\
\text { (mag) }\end{array}$ & $\begin{array}{l}J_{\text {Stetson }}{ }^{f} \\
\text { opt-IR }\end{array}$ & SED Slope $^{g}$ & $\begin{array}{l}\tau_{\mathrm{opt}}^{\mathrm{h}} \\
\text { (days) }\end{array}$ \\
\hline CSIMon-000119* & $06412100+0933361$ & 223985987 & K6 & -10.6 & 2.9 & 0.27 & 2.30 & -1.42 & 1.6 \\
\hline CSIMon-000242 & $06411185+0926314$ & 602079796 & K7 & -5.4 & 0.4 & 0.14 & 1.16 & -1.39 & 5.3 \\
\hline CSIMon-000346 & $06410908+0930090$ & 603402478 & K7 & -27 & 4.1 & 0.28 & 0.78 & -0.63 & 1.5 \\
\hline CSIMon-000425 & $06411668+0929522$ & 616943882 & K5 & -6.3 & 2.4 & 0.30 & 1.31: & -1.02 & 1.1 \\
\hline CSIMon- $000457^{*}+$ & $06410673+0934459$ & 616919789 & G6 & -49.4 & $>10$ & 0.18 & 0.97: & -0.18 & 3.7 \\
\hline CSIMon-000491+ & $06405616+0936309$ & 616895873 & K3 & -67.2 & 5.2 & 0.50 & 0.41: & -0.63 & 4.6 \\
\hline CSIMon-000577 & $06414382+0940500$ & 616895846 & K1 & -5.9 & 2.9 & 0.10 & 0.72 & -1.55 & 1.3 \\
\hline CSIMon-000613+ & $06410577+0948174$ & 616849463 & K6.5 & -30.1 & 2.1 & 0.14 & 0.92: & -1.51 & 1.4 \\
\hline CSIMon-000636+ & $06404884+0943256$ & 616872578 & M0 & -15.5 & 5.4 & 0.49 & 1.63 & -0.57 & 1.2 \\
\hline CSIMon-000795* & $06411257+0952311$ & 500007115 & $\mathrm{GVe}$ & -35.3 & 2.5 & 0.22 & $\cdots$ & -1.55 & 0.5 \\
\hline CSIMon-000985 & $06401515+1001578$ & 223969098 & G8* & -9.1 & $>10$ & 0.51 & 1.99 & -1.12 & 0.7 \\
\hline CSIMon-001054*+ & $06403652+0950456$ & 400007538 & M2 & -21.1 & 2.0 & 0.15 & 0.53 & -1.29 & 0.8 \\
\hline CSIMon-001061 & $06402416+0934124$ & 616919835 & M0 & -33.0 & 3.5 & 0.33 & 1.99 & -1.06 & 0.8 \\
\hline CSIMon-001234+ & $06401113+0938059$ & 223968039 & K6 & -52.9 & 1.4 & 0.60 & 1.55 & -1.39 & 1.2 \\
\hline CSIMon-001267 & $06403431+0925171$ & 616944066 & $\mathrm{G} 5^{*}$ & -15.0 & 5.4 & 0.25 & $\cdots$ & -0.47 & 1.1 \\
\hline CSIMon-006491 & $06392550+0931394$ & 616920065 & $\mathrm{~K} 4^{*}$ & -6.2 & 1.7 & 0.29 & 1.64 & -1.52 & 0.5 \\
\hline CSIMon-006930 & $06390409+0916139$ & 223948224 & $\cdots$ & $\cdots$ & $\cdots$ & 0.18 & $\cdots$ & $\cdots$ & 0.4 \\
\hline
\end{tabular}

Notes.

${ }^{\text {a }}$ The CSIMon IDs are our internal naming scheme for stars in the field of NGC 2264—see Cody et al. (2014) and http://irsa.ipac.caltech.edu/data/SPITZER/ CSI2264. In the main text of this paper, we omit the "CSI" and the leading zeros in the object names for brevity. ${ }^{*}=$ synoptic VLT spectra from 2011 available; $+=$ synoptic VLT spectra from 2013 available.

${ }^{\mathrm{b}}$ CoRoT identification number. Three stars were observed in both 2008 and 2011, but had different ID numbers in 2008-the 2008 CoRoT ID number for those three stars are 500007221 (Mon-242), 500007369 (Mon-457), and 500007548 (Mon-1267).

${ }^{\mathrm{c}}$ Spectral types marked with an asterisk have their types estimated from the dereddened CFHT photometry and the Pecaut \& Mamajek (2013) young star relation between effective temperature and spectral type; see Venuti et al. (2014). The H $\alpha$ equivalent widths for these stars come from our spectra. All the other spectral types and $\mathrm{H} \alpha$ equivalent widths are from Dahm \& Simon (2005), except for Mon-636 where the spectral type is from Makidon et al. (2004).

${ }^{\mathrm{d}}$ FR4.5 is an estimate of the ratio of the flux from the disk to the flux from the stellar photosphere at $4.5 \mu \mathrm{m}$, based on all the available broad-band photometry and the published spectral types. Because the SED models do not include veiling, these values are likely lower limits.

${ }^{\mathrm{e}}$ The 10\%-90\% amplitude of the CoRoT light curves. When we have data for both 2008 and 2011, the CoRoT amplitude corresponds to the epoch plotted in Figure 2 and Figure 3.

${ }^{\mathrm{f}}$ Stetson index between the CoRoT 2011 light curve and the IRAC [4.5] light curve. A colon following the value indicates we only have a small number of IRAC photometry points, making the value uncertain.

${ }^{\mathrm{g}}$ Spectral energy distribution (SED) slope from 2 to $8 \mu \mathrm{m}$, derived as in Rebull et al. (2014).

${ }^{\mathrm{h}}$ Optical light curve timescale, as discussed in Section 5.4.

data we obtained with CoRoT, Spitzer, VLT/FLAMES, Canada-France-Hawaii Telescope (CFHT) and the USNO $1.0 \mathrm{~m}$ telescopes, which we summarize in Table 1 .

In addition to the synoptic data, we also use several single epoch data sources in order to help characterize the overall properties of the NGC 2264 members. These data sets include:

1. Single epoch ugri imaging of the entire cluster, with standard Sloan Digital Sky Survey filters, obtained using Megacam on the CFHT during two observing runs in 2010 and 2012. These data are presented in detail in
Venuti et al. (2014). We also make use of single-epoch UBVRI data from Sung et al. (2008).

2. Spitzer IRAC 3.6, 4.5, 5.8 and $8.0 \mu \mathrm{m}$ and MIPS $24 \mu \mathrm{m}$ single epoch imaging photometry of the entire cluster obtained early in the cryogenic mission, and reported in Sung et al. (2009) and Teixeira et al. (2012).

3. Single epoch FLAMES spectra for many of the NGC 2264 members, originally obtained as part of the ESO-Gaia program (Randich et al. 2013). We downloaded the raw spectra from the ESO-Gaia archive for these stars, and produced our own calibrated spectra. 
Table 3

Photometric Data for the Stars of Table 2

\begin{tabular}{|c|c|c|c|c|c|c|c|c|c|c|c|c|}
\hline Mon ID & $u$ & $g$ & $r$ & $i$ & $J$ & H & $K_{s}$ & {$[3.6]^{a}$} & {$[4.5]^{\mathrm{a}}$} & {$[5.8]$} & {$[8.0]$} & [24] \\
\hline CSIMon-000119 & 17.819 & 15.653 & 14.507 & 14.049 & 12.433 & 11.631 & 11.188 & 10.451 & 10.110 & 9.885 & 9.159 & 6.308 \\
\hline CSIMon-000242 & $\ldots$ & $\ldots$ & $\ldots$ & $\ldots$ & 12.210 & 11.521 & 11.255 & 10.823 & 10.700 & 9.572 & $\ldots$ & $\ldots$ \\
\hline CSIMon-000346 & 19.207 & 17.354 & 15.943 & 14.880 & 12.945 & 11.963 & 11.543 & 10.266 & 9.786 & 9.198 & 8.449 & $\ldots$ \\
\hline CSIMon-000425 & 17.190 & 14.737 & 13.626 & 13.782 & 11.268 & 10.500 & 10.071 & 9.327 & 8.956 & 8.553 & 7.430 & 4.306 \\
\hline CSIMon-000457 & 19.016 & 16.545 & 14.929 & 14.884 & 11.761 & 10.279 & 9.234 & 7.949 & 7.350 & 6.700 & 5.433 & 1.608 \\
\hline CSIMon-000491 & 18.346 & 16.485 & 14.979 & 14.726 & 11.892 & 10.816 & 10.047 & 8.941 & 8.500 & 7.921 & 6.910 & 3.969 \\
\hline CSIMon-000577 & 16.510 & 14.613 & 13.770 & 13.416 & 12.047 & 11.255 & 10.692 & 10.072 & 9.810 & 9.566 & 8.842 & 5.951 \\
\hline CSIMon-000613 & 16.996 & 15.608 & 14.547 & 13.835 & 12.020 & 11.260 & 10.802 & 10.135 & 9.838 & 9.617 & 8.891 & 6.076 \\
\hline CSIMon-000636 & 20.389 & 18.321 & 16.735 & 15.832 & 13.334 & 12.282 & 11.706 & 10.476 & 9.924 & 9.402 & 8.509 & 6.306 \\
\hline CSIMon-000795 & 16.069 & 14.487 & 13.574 & 13.202 & 11.494 & 10.686 & 10.205 & 9.364 & 9.056 & 8.844 & 8.397 & 5.950 \\
\hline CSIMon-000985 & 21.404 & 18.918 & 17.379 & 15.696 & 14.548 & 13.252 & 12.353 & 10.933 & 10.625 & 10.425 & 9.890 & 7.187 \\
\hline CSIMon-001054 & 17.742 & 16.890 & 15.885 & 15.075 & 12.934 & 12.141 & 11.707 & 11.066 & 10.678 & 10.258 & 9.548 & 5.866 \\
\hline CSIMon-001061 & 18.131 & 17.396 & 16.366 & 15.614 & 13.471 & 12.521 & 11.839 & 11.116 & 10.648 & 10.250 & 9.316 & 6.445 \\
\hline CSIMon-001234 & 19.672 & 17.077 & 15.566 & $\cdots$ & 12.955 & 12.116 & 11.615 & 10.907 & 10.596 & 10.318 & 9.530 & 6.650 \\
\hline CSIMon-001267 & 22.738 & 19.800 & 17.740 & 17.260 & 14.651 & 13.175 & 12.249 & 10.940 & 10.389 & 9.790 & 8.932 & 6.329 \\
\hline CSIMon-006491 & 20.073 & 18.226 & 16.746 & 15.808 & 13.770 & 12.861 & 12.384 & 11.731 & 11.441 & 11.151 & 10.525 & 7.862 \\
\hline CSIMon-006930 & 19.739 & 17.221 & 15.902 & 15.125 & 13.627 & 12.893 & 12.677 & [12.36] & [12.16] & $\cdots$ & $\cdots$ & $\cdots$ \\
\hline
\end{tabular}

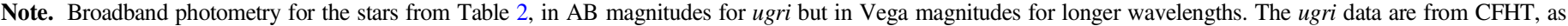

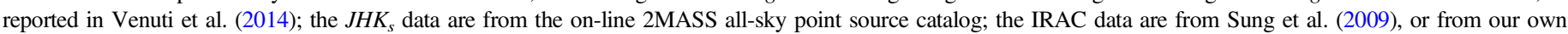

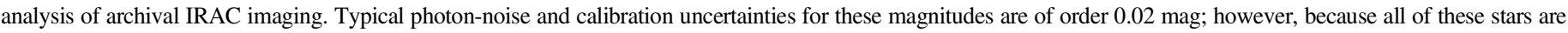

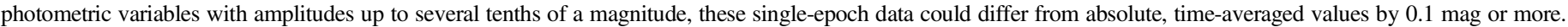

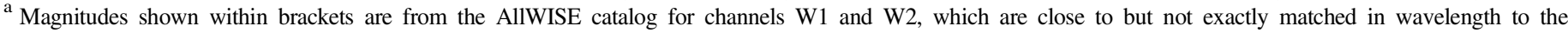
corresponding IRAC channels.

We have used the VLT/FLAMES spectra to analyse $\mathrm{H} \alpha$ profiles, spectral veiling, projected rotational velocities and emission line FWHM as diagnostics of accretion and the geometry of the CTTS disks. Details of this analysis are generally discussed in the Appendix.

\section{THE YSOs IN NGC 2264 WITH STOCHASTIC LIGHT CURVES}

As noted in Section 1 and in Cody et al. (2014), the stochastic light curve class includes stars whose CoRoT photometry shows little or no periodic signature and for which the light curve has no significant preference for upward excursions or downward excursions (they appear symmetric when reflected about their median level). Quantitatively, this corresponds to $Q>0.61$ and $-0.25<M<0.25$ using the Cody et al. (2014) metrics, where the $Q$ metric is a measure of the light curve periodicity (or stochasticity) and the $M$ metric quantifies the degree of symmetry of the light curve relative to its median level. Light curves for prototypes of the stochastic class are shown in Figure 18 of Cody et al. (2014). Those light curves resemble model light curves produced by damped random walk processes (Findeisen et al. 2015), which is the reason for adopting "stochastic" as the descriptive name for this class.

Because Cody et al. (2014) considered only the 2011 CoRoT data (and not the 2008 campaign) and because our membership list has evolved slightly since that paper was written, we have conducted a thorough review of all of our data in order to construct the list of stochastic stars to analyse in this paper. We also have chosen to add two additional criteria in order to make the sample more characterizable and more "pure." First, we require that the full amplitude of the CoRoT light curve be greater than $0.1 \mathrm{mag}$; this criterion makes it more likely that we will be able to detect correlations between the CoRoT light curve shape and other data. Second, we have attempted to exclude stars whose light curve morphology appears to be better described as a mix of similar amplitude accretion bursts and extinction dips. Light curves with features arising from a mixture of mechanisms is both possible and expected; however, we have associated specific physical mechanisms already with accretion burst signatures and extinction dips. Separating truly random walk light curve morphologies from this kind of mixed mechanism morphology is admittedly subjective, and we probably have not been completely successful. However, because our goal is not to identify a complete set of objects but is instead to identify the physical mechanism driving this light curve morphology, we believe this process is acceptable.

Table 2 presents the list of NGC 2264 YSOs whose light curve satisfy our criteria. The table provides our internal name for the star, as well as the 2MASS and CoRoT designations. In addition, we provide spectral type, $\mathrm{H} \alpha$ equivalent width, light curve amplitude in the optical and infrared, an optical-IR Stetson index (Stetson 1996), a spectral energy distribution (SED) slope, and a timescale (see Section 5.4). The suite of single-epoch broadband photometry we have for each of these stars is provided in Table 3; most of these data have appeared previously in Venuti et al. (2014) and Cody et al. (2014) — we repeat their values here in order to allow readers to easily associate specific stars to specific points in the color-color and color-magnitude plots we show. The CoRoT light curves for each of these stochastic light curve stars are provided in Figures 2 and 3. There are of order 175 CTTS with good CoRoT light curves; with seventeen stars in the stochastic light curve class, the frequency of occurrence for light curves of this type and our selection criteria is of order 10\% (versus 13\% in Cody et al. 2014 using their slightly different selection criteria and their slightly different membership list). 

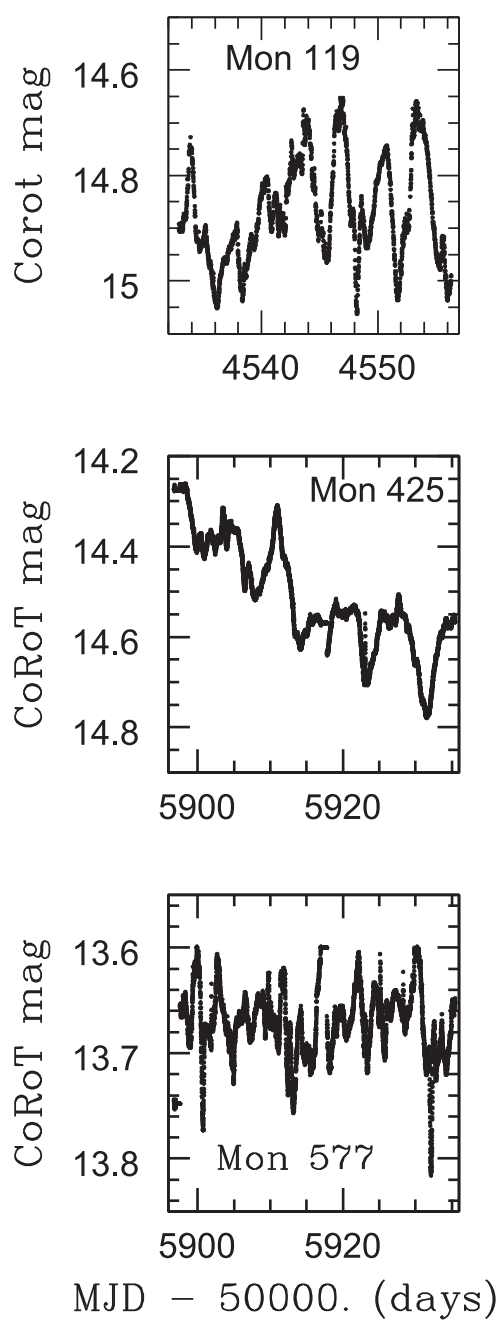
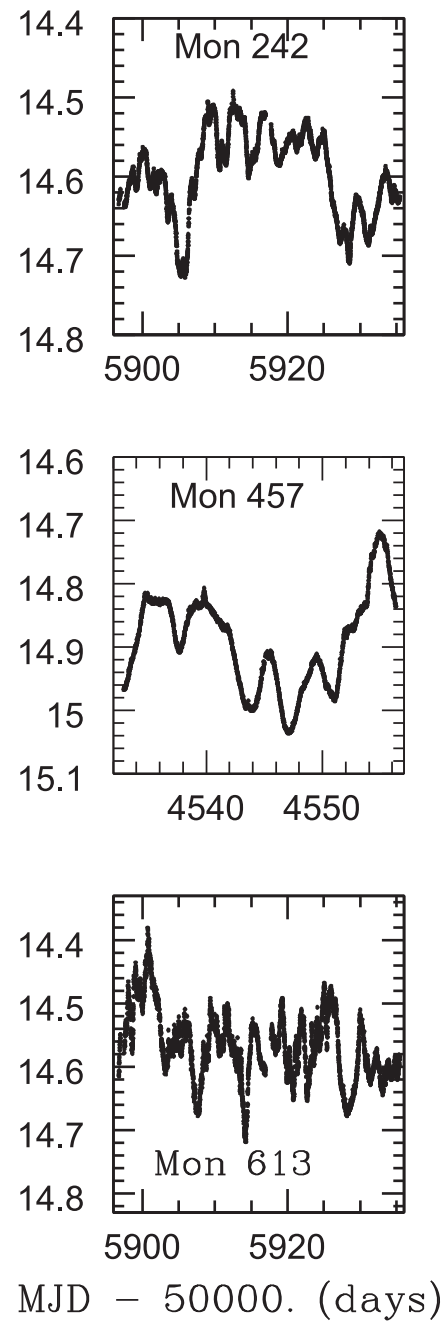
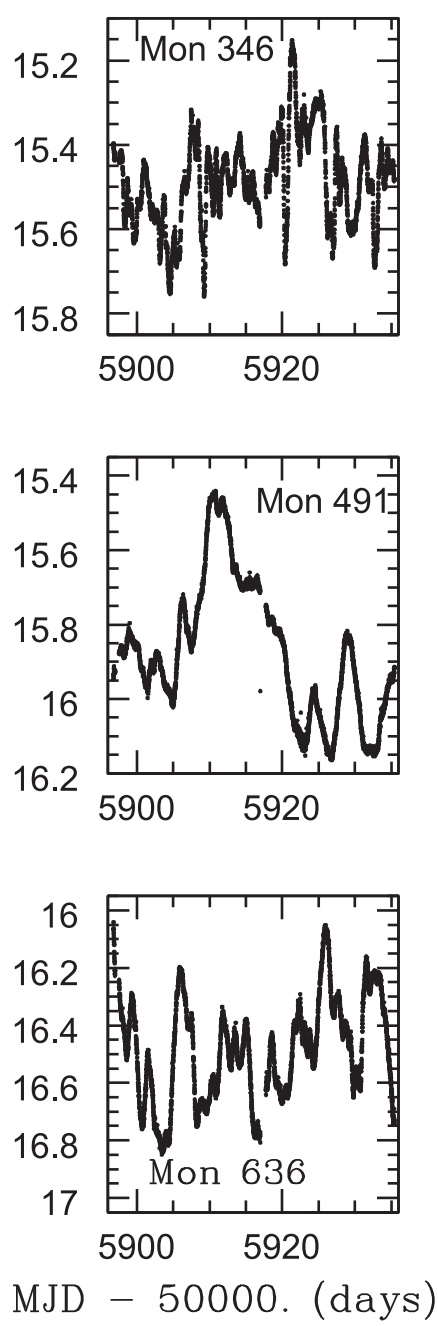

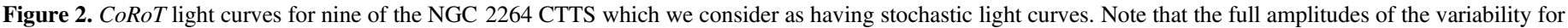
these stars is generally in the 10\%-30\% range, which is small compared to the stars with variable-extinction dominated light curves (McGinnis et al. 2015).

Though we have not required the stars in Table 2 to have $Q$, $M$ metrics that satisfy the Cody et al. (2014) criteria, most of them do so, as is illustrated in Figure 4. Our sample includes two stars whose light curve metrics fall slightly outside the nominal stochastic-class $Q M$ box boundary. One of them (Mon-457) has CoRoT light curves in both 2008 and 2011; the 2008 light curve falls within the $Q M$ box, whereas the 2011 light curve falls slightly below the $Q M$ lower boundary. The other star whose light curve is slightly below the lower $Q M$ boundary is Mon-242; we include it in Table 2 simply because its light curve morphology seems best matched to this class.

Seven additional stars also satisfy the $Q M$ criteria for having stochastic light curves, but we believe they are better classified as belonging to other groups. The light curves for these seven stars are shown in the Appendix in Figure 20. The stars in Figure 20 include three stars (Mon-11, Mon-510 and Mon-996) which we classified as having accretion-burst dominated light curves and included in Stauffer et al. (2014); two additional accretion burst dominated stars (Mon-766 and Mon-1048), one star which we believe is best interpreted as having a light curve dominated by a combination of accretion bursts and flux dips due to variable extinction (Mon-1294), and one star (Mon-774) whose 2011 light curve we attribute to aperiodic extinction dips. Other interpretations could be made for the 2011 Mon-
774 light curve; our choice was strongly influenced by the fact that its 2008 light curve was clearly that of an AA Tau analog (periodic, deep, broad flux dips due to variable extinction) and that stars with AA Tau-type light curves often switch to aperiodic flux dip dominated light curves at other epochs (McGinnis et al. 2015).

While the $Q$ and $M$ statistics for the stars in Figure 2 and Figure 3 assign them to the same morphological light curve class, at first glance their light curves nevertheless appear somewhat heterogeneous. In significant part, that is because the characteristic timescales for their variations differ (see Section 5). Specifically, some of the stars have quite short characteristic timescales (e.g., Mon-346, 577, 985 and 6491), whereas others have relatively long timescales (e.g., Mon-425, $457,491)$. In order to better illustrate the similarity of their light curves, Figure 5 shows the light curves of two of the stars with short timescales in an expanded view. With the $x$-axis expanded roughly five times in scale, the light curves for these two stars (Mon-346 and Mon-985) look much more similar to the light curves for the long timescale members of the class.

Before proceeding to our discussion of the CoRoT light curves for the stochastic stars and the probable physical mechanism causing their optical variability, it is useful to consider the physical properties of these stars as derived from 

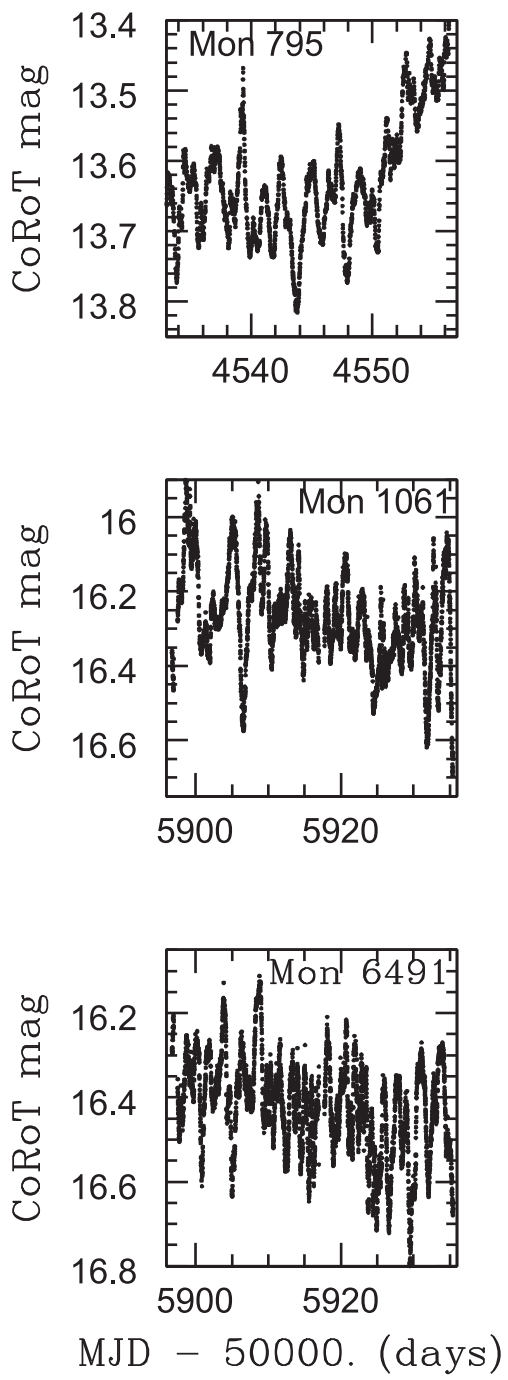
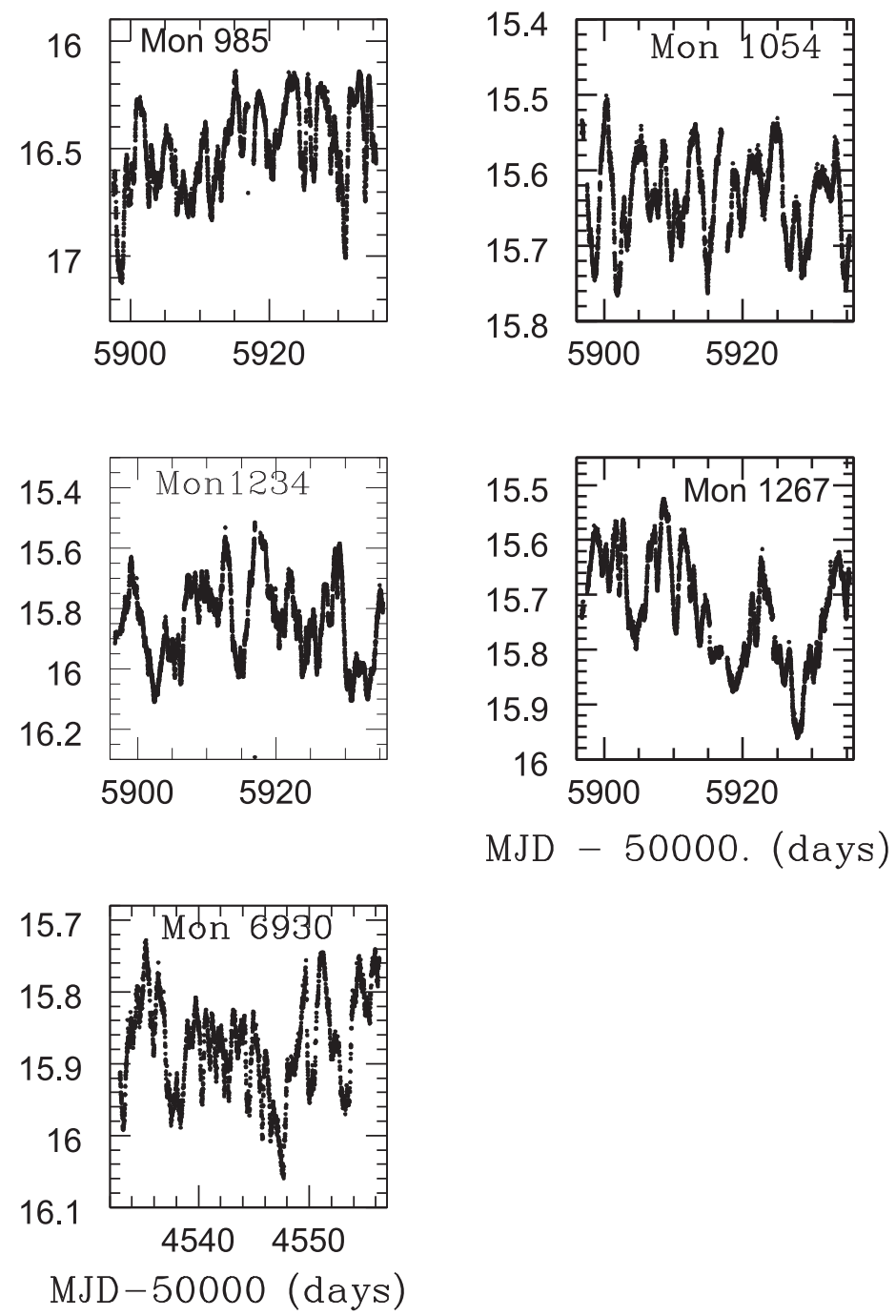

Figure 3. CoRoT light curves for the other seven NGC 2264 CTTS which we consider as having stochastic light curves. their mean observed colors and spectral types. If these stars differ in some significant aspect from the other CTTS in NGC 2264, that could help us interpret their photometric variability. Toward this end, we provide four diagnostic plots: a $g$ versus $(g-i)$ color-magnitude diagram (CMD); a $(u-g)$ versus $(g-r)$ color-color plot; a $(J-H)$ versus $\left(H-K_{s}\right)$ near-IR color-color plot, and the IRAC [3.6] - [4.5] versus [5.8] - [8.0] color-color plot. Our conclusions from these plots and the data listed in Tables 2 and 3 are:

(1) From the near-IR color-color diagram (Figure 6(a)), we infer that on average the stochastic stars have relatively small extinctions, comparable to, but probably on average somewhat larger than those for the accretion burst and variable extinction classes (a list of the accretion burst and variable extinction stars plotted in these four figures and used elsewhere in the paper is provided in Table 5). This agrees with extinction estimates derived from combining the observed $(V-I)$ colors (Sung et al. 2008), published spectral types (Table 2 and Cody et al. 2014), and standard colors for young stars from Pecaut \& Mamajek (2013), where the median $E(V-I)$ colors are $0.42,0.05$ and 0.15 for the stochastic, accretion burst and variable-extinction classes, respectively. The derived $E(V-I)$ for the accretion burst stars is probably an underestimate because their $(V-I)$ colors are significantly influenced by hot spots, while the $E(V-I)$ colors of the variable extinction stars may be underestimated if scattered light from the disk contributes significantly to their optical flux. The three stochastic stars with the largest $\left(H-K_{s}\right)$ colors (Mon-457, 491, and 985) also have the largest disk to photosphere contrast ratios at $4.5 \mu \mathrm{m}$ (see Table 2 , column 7 ), demonstrating that their light is disk-dominated in the IR.

(2) From the optical CMD (Figure 6(b)), we conclude that the stochastic stars are not, on average, displaced "vertically" relative to the other CTTS groups nor relative to the weak-lined T Tauri (WTTS) sample. If the CMD can be used to infer relative ages, this indicates no significant age difference between these stars despite their quite different light curve morphologies. As shown in Table 2, the stochastic class includes YSOs with spectral types spanning the entire $G$ to $\operatorname{mid}-M$ range. The stochastic and variable extinction classes dominate the upper-left portion of the CMD, indicating they probably include a larger fraction of high mass CTTS compared to the accretion burst class. Comparison of the spectral type 


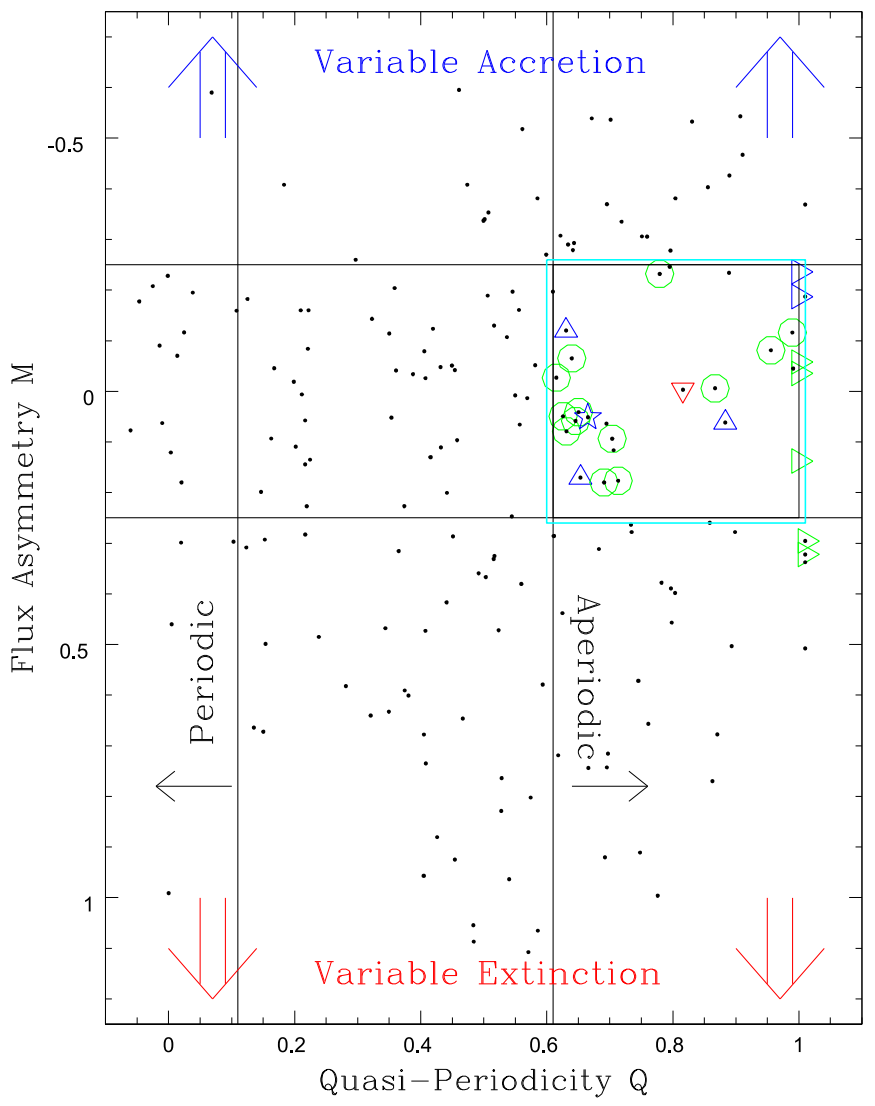

Figure 4. Plot derived from the CoRoT light curves for CTTS in NGC 2264 illustrating the quantitative light curve morphology classification technique, and the location of the stars studied in this paper within that plot (the region inside the cyan box). $Q<0.11$ corresponds to periodic light curves (typically reflective of rotational modulation of a stellar photosphere mottled by relatively stable, cold spots); $0.11<Q<0.61$ indicates quasi-periodicity (Cody et al. 2014), typically corresponding to light curves showing periodic structure whose waveform evolves significantly from period to period as often true for AA Tau-analogs; and $Q>0.61$ indicates stars with little or no periodic signature. Blue triangles denote stars with accretion burst light curves located within the stochastic region (cyan box); blue stars have light curves with both accretion bursts and narrow flux dips; the red triangle marks a star with a variable extinction light curve; and green circles and triangles are stars we classify as having stochastic light curves (with the triangles being those with only limits on $Q$ ).

distributions for the three classes confirms this difference (see Section 5.2).

(3) Figure 7(a), the $(u-g)$ versus $(g-r)$ diagram, has been used as an accretion diagnostic in Venuti et al. (2014). Young stars without on-going accretion form an inverted $\mathrm{V}$ in this diagram, with early $\mathrm{G}$ stars located near $g-r=0.5, u-g=2.0$, and with both colors becoming redder to later spectral types until about M0 (at $g-r \sim 1.4, u-g \sim 2.8$ ). Later $M$ spectral types retain $g-r \sim 1.4$ but become bluer in $u-g$, presumably due to enhanced chromospheric emission. Reddening shifts stars toward the upper right in the diagram; accretion primarily shifts stars down but also slightly to the left. The diagram shows that, on average, the stochastic stars have smaller UV excesses than the stars with accretionburst dominated light curves, but comparable UV excesses as for the variable extinction sample. A Student- $T$ test yields a less than $10^{-4}$ probability that the accretion burst stars are drawn from the same parent
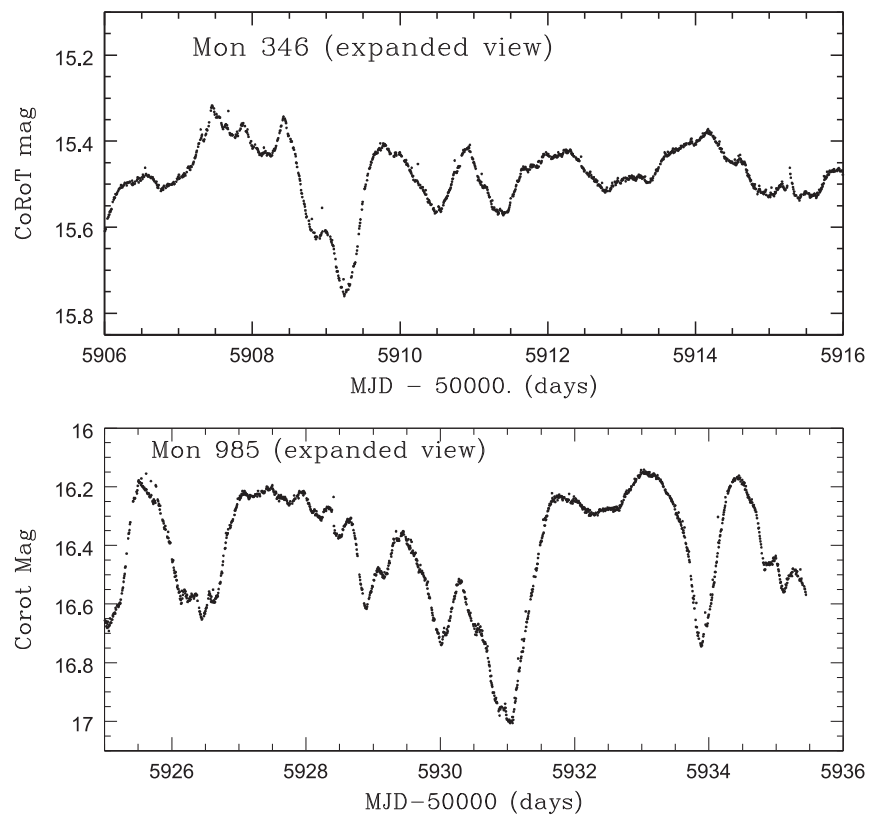

Figure 5. CoRoT light curves for two members of the stochastic light curve class, zooming in on a 10 day section of their data, in order to illustrate better the similarity in morphology to other members of the class with longer timescale variations.

population as the other two classes, but finds no significant difference between the stochastic and variable extinction classes. Also in support of these conclusions, the median $\mathrm{H} \alpha$ equivalent widths for the stochastic, accretion burst and variable extinction classes are -18 , -68 , and $-16 \AA$, respectively.

(4) Figure 7(b) shows the IRAC two-color diagram for the same set of stars. All of the stochastic stars fall within the Class II box defined in Allen et al. (2004) or slightly redward of that box. This suggests that their inner disks are similarly dusty, on average, as the accretion-burst dominated class (which have a very similar distribution of points in this diagram). The stochastic stars have redder IRAC colors, on average, compared to the stars in the variable extinction light curve class (a Student-T test yields a $1 \%$ probability that the two classes have the same parent population colors for each axis). Comparison of the mean SED slopes from 2 to $8 \mu$ m yields a similar result, with mean slopes of $-1.08,-1.21$, and -1.54 (and statistical uncertainties of about 0.10 for each of these values) for the stochastic, accretion burst and variable extinction classes, respectively.

\section{VARIABLE ACCRETION AS THE PHYSICAL MECHANISM TO EXPLAIN THE STOCHASTIC LIGHT CURVES}

In the Herbst et al. (1994) taxonomy, stars in their Type II light curve category would generally map into Cody's stochastic class. Herbst et al. advocated that the most probable physical mechanism to explain their Type II light curves was variable hot spots possibly accompanied by rotational modulation bringing those hot spots into or out of the visible hemisphere. We agree with that assessment. As noted by Herbst et al., if this is the case, the light curve modulation 

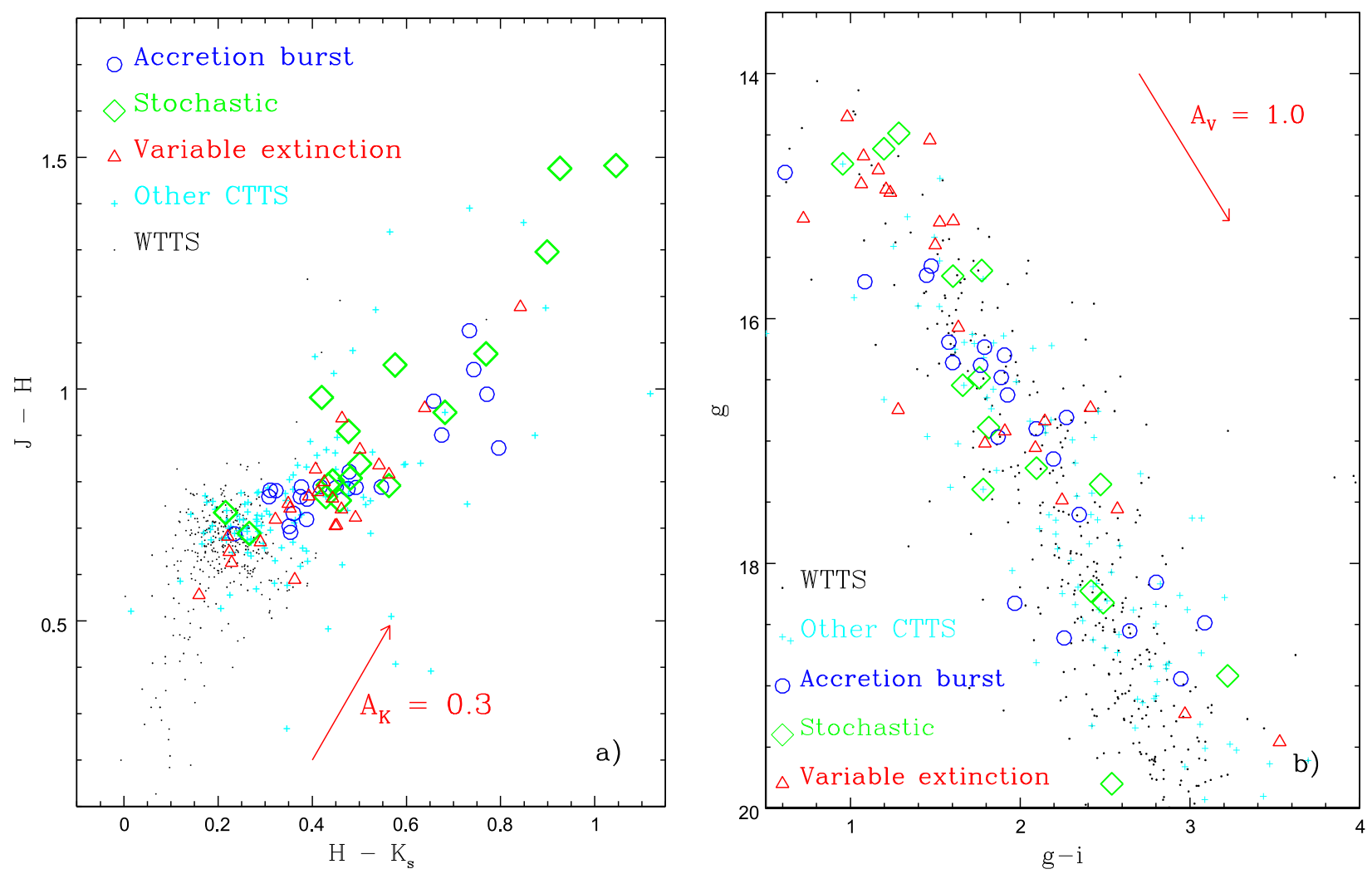

Figure 6. (a) Near-IR color-color diagram for stars with stochastic CoRoT light curves, comparing those stars to CTTS whose light curve morphologies we ascribe to variable extinction (AA Tau and Ap classes in McGinnis et al. 2015) and to stars whose light curves show short-duration accretion bursts (Stauffer et al. 2014). Small black dots are WTTS NGC 2264 members; small cyan plus signs are CTTS that are not members of the three light curve classes highlighted in the plot. The stochastic light curve stars have locations in this diagram that are not greatly different, on average, compared to the other light curve classes, but are somewhat weighted to redder colors (more extinction or more warm dust); and (b) an optical color-magnitude diagram for the same set of stars. This diagram shows no obvious systematic age differences between the stochastic light curve stars and the members of the other two CTTS light-curve classes or the WTTS.

should be accompanied by changes in spectral veiling, resulting in photospheric absorption lines having smaller equivalent widths when the hot spot contribution to the optical light is greater. For the stochastic stars for which we have synoptic VLT spectroscopy, we will show that they do exhibit variable veiling consistent with the amplitude of the optical variability shown in the CoRoT light curves.

The linkage of changes in the spectral veiling to the optical light curve morphology is made difficult because most of our spectroscopy was obtained after the CoRoT photometric campaigns. Also, in some cases, the only photospheric absorption line we can measure accurately is the lithium 6708 doublet, whose equivalent width could be affected by other processes (Basri et al. 1991; Barrado et al. 2001; Baraffe \& Chabrier 2010). Our analysis therefore proceeds as follows: (a) for the brighter stars with synoptic VLT spectra, we show that their spectra do show significant veiling variability as measured by several atomic absorption features, and the variability shown by the lithium doublet is well-correlated with the veiling changes; (b) for all of the stochastic stars where we can measure variations in the lithium absorption strength, that variability correlates well with changes in the strength of the He I $6678 \AA$ emission line-an accretion diagnostic; (c) the inferred continuum level changes needed to explain the veiling variability is consistent with the measured CoRoT light curve amplitudes; (d) the mean veiling of the stochastic stars is small, consistent with their UV excesses; and (e) the UV variability seen in the stochastic stars, as measured by the slope shown in a delta $(r)$ versus delta $(u-r)$ CMD is also best explained by variable hot spots (see also Venuti et al. 2015).

\subsection{Veiling and Accretion Variability}

For four of the stars with stochastic light curves, we have synoptic VLT spectra of good enough quality to accurately measure equivalent widths for $\mathrm{Li}$ I 6708 and at least one other photospheric absorption line. These observations show that there are large variations in the absorption line equivalent widths (veiling variability) over the time period of the VLT campaigns and that the lithium strength variations track well the variations in the other absorption features. Figure 8 shows the equivalent width data for these four stars, along with similar data for two of the accretion burst stars. The veiling variability is a natural consequence of a varying hot spot contribution to the star's continuum flux and is not expected for any of the other mechanisms that are known to drive significant photometric variability in low mass YSOs (e.g., cold spots or variable extinction).

For the four stars above, plus one additional stochastic star, we have synoptic VLT spectra from which we can accurately measure the $\mathrm{Li}$ I 6708 equivalent width and for which the $\mathrm{He}$ I 6678 line is sometimes in emission. The $\mathrm{He}_{\mathrm{I}} 6678$ line is present in high-resolution spectra for many CTTS, sometimes as a broad feature possibly associated with a hot wind (Beristain et al. 2001), and sometimes as a narrow (FWHM 

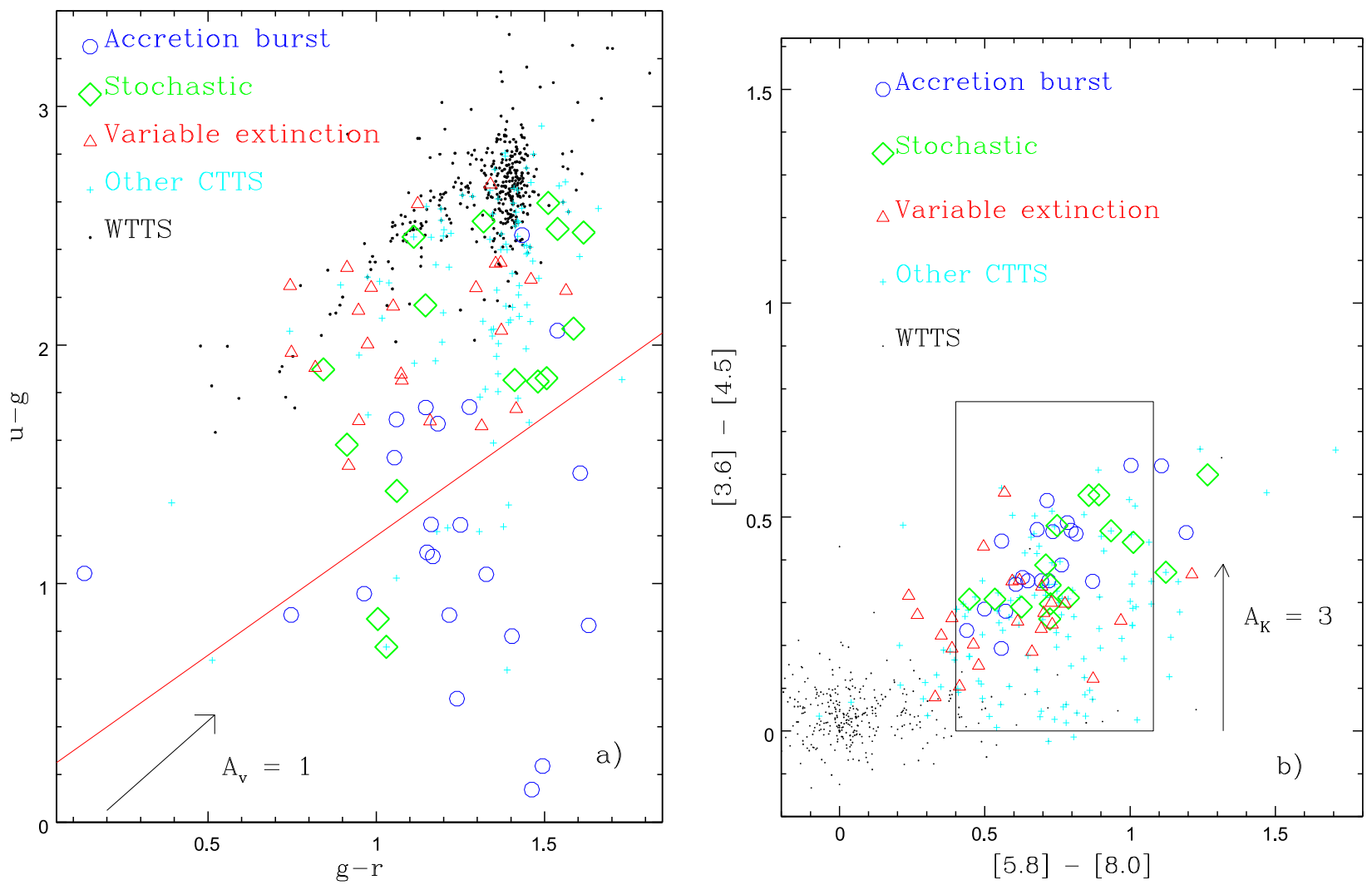

Figure 7. (a) $u-g$ vs. $g-r$ color-color diagram for stars with stochastic CoRoT light curves, comparing those stars to CTTS whose light curves we ascribe to variable extinction and to stars whose light curves show short-duration accretion bursts. Small black dots are WTTS; small cyan plus signs are CTTS that are not members of the three light curve classes highlighted in the plot. The diagonal red line is a rough boundary between stars with weak and strong UV excesses (Venuti et al. 2014). Note that one of the stochastic stars-Mon-1267-falls outside the limits of the plot at $g-r=2.06, u-g=2.94$. The locus of points for the stochastic light curve stars in this diagram is similar to that for the variable extinction stars (and has much lower UV excesses on average than the accretion burst stars); and (b) mid-IR color-color diagram for the same set of stars. The rectangular box encloses the region dominated by Class II sources as defined in Allen et al. (2004). In this diagram, the stochastic light curve stars have IR colors similar to those for the accretion burst stars, and distinctly redder, on average, compared to the stars in the variable extinction class.

$\sim 36 \mathrm{~km} \mathrm{~s}^{-1}$ ), approximately Gaussian feature, usually ascribed to hot gas created in the shock where the accretion flow impacts the stellar photosphere (Johns-Krull et al. 2013). Our stochastic light curve stars with VLT spectra only show the narrow He I emission feature. Therefore, we would expect stronger He I emission to correlate with stronger accretion flux, and the $\mathrm{He}_{\mathrm{I}}$ emission and $\mathrm{Li}$ I 6708 absorption strengths should correlate for stars where accretion variability is strong. Figure 9 shows that this correlation is indeed present for all five stars with stochastic light curves where we have the data to make this test (the figure also includes data for Mon-945, a star with an accretion-burst dominated light curve, to illustrate how this diagram should appear if accretion drives the variability).

Figures 8 and 9 prove that at least these specific members of the stochastic light curve class have optical brightnesses that vary with time due to variable accretion. However, this does not necessarily require that their light curve shapes and amplitudes are primarily due to variable accretion luminosity -other mechanisms (such as variable extinction) could cause optical brightness variations that in principle could be larger than the accretion-driven variations. If our spectroscopy and photometry were simultaneous, we could directly compare the continuum flux variations inferred from the spectroscopy with the light curve amplitudes-however, our CoRoT data are from 2008 and 2011, whereas the majority of our spectroscopy is from 2013. Even for those stars where we have 2011 multiepoch spectroscopy, the overlap in time with the CoRoT campaign is small, and we therefore cannot make a useful direct comparison. However, we can make a statistical test-is the inferred range in accretion flux at $6700 \AA$ derived from the veiling data (mostly from 2013) consistent with the measured amplitude in the CoRoT light curves (mostly from 2011)? Figure 10 shows this comparison for the stochastic stars for which we have multi-epoch VLT data. There is considerable scatter in the plot, but the data are consistent with the variable continuum inferred from the spectroscopy matching the variable continuum measured in the light curves-supporting the conclusion that variable accretion is the dominant cause of the optical variability.

Finally, having argued that the lithium equivalent widths for CTTS can be used as a relative veiling (and hence accretion) indicator, we can also use the mean lithium equivalent widths for the stochastic light curve stars to estimate their mean veiling levels. In Table 4, we provide mean lithium equivalent widths for the stochastic light curve stars, either measured from our multi-epoch VLT/FLAMES data, or in six cases, as measured from single-epoch VLT/ FLAMES spectra obtained by the ESO-Gaia project (Randich et al. 2013). We also provide an estimated maximum photospheric lithium equivalent width for that spectral type, 

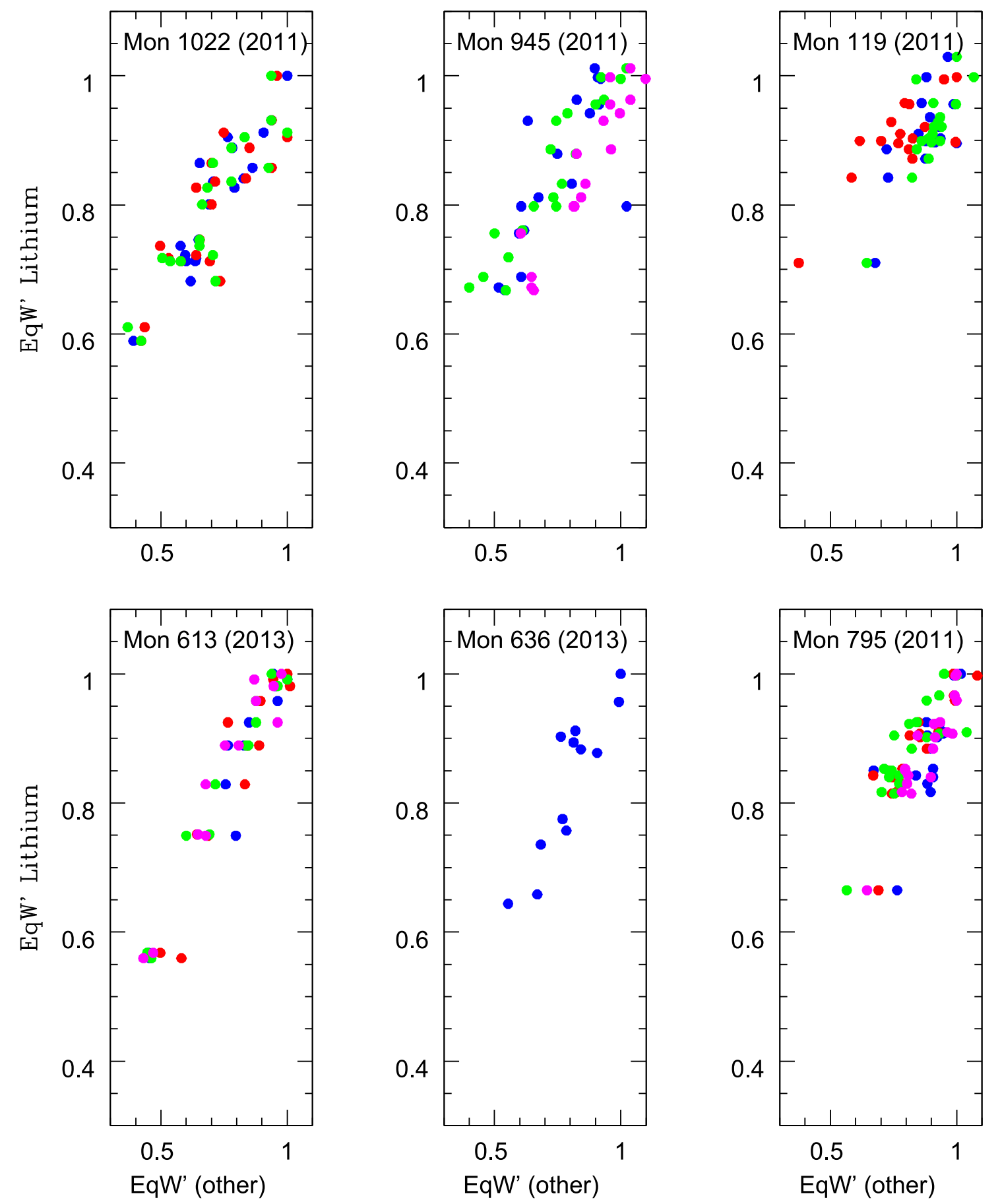

Figure 8. Correlation between the equivalent width for the Li I $6708 \AA$ A doublet and that of other absorption lines present in the same FLAMES echelle order, for the four stochastic stars with high enough $\mathrm{S} / \mathrm{N}$ in their spectra to measure these features accurately. The equivalent widths at each epoch have been divided by the maximum equivalent width for that feature, in order to allow data for several features to be over-plotted. Also shown for comparison are the same plot for Mon-1022 and Mon-945, NGC 2264 members whose light curves are dominated by accretion bursts (Stauffer et al. 2014). Each color corresponds to a different absorption feature (blue: $6463 \AA, \mathrm{Ca}$ I $+\mathrm{Fe}$ I; red: 6492-6500 $\mathrm{A}$, a blend of $\mathrm{Ca}$ I, Fe I and Fe II lines; and green: $6594 \AA$, an Fe I doublet). The good correlation and large range in equivalent widths shown for these stars shows that their spectra have significant contribution from a light source with relatively smooth continuum and variable strength, best explained as due to hot spots.

based on a fit to the upper-envelope of the lithium equivalent widths for all YSOs in NGC 2264 for which we have FLAMES spectra. This allows us to derive an upper limit to the veiling at $6700 \AA$ for each of these stochastic stars. It can be seen that the mean lithium equivalent widths for the stochastic stars differ only slightly from their expected photospheric values, suggesting veilings of generally $10 \%$ or less. The median lithium veiling from Table 4 for the stochastic stars is 0.17 ; for reference, the median veiling for the accretion burst and variable extinction classes are 0.36 and 0.17 . The average veiling we derive for the stochastic stars therefore agrees with the conclusion drawn from their UV excesses (Figure 7(a)) that stars of this light curve class have low to moderate accretion rates (significantly lower than stars 

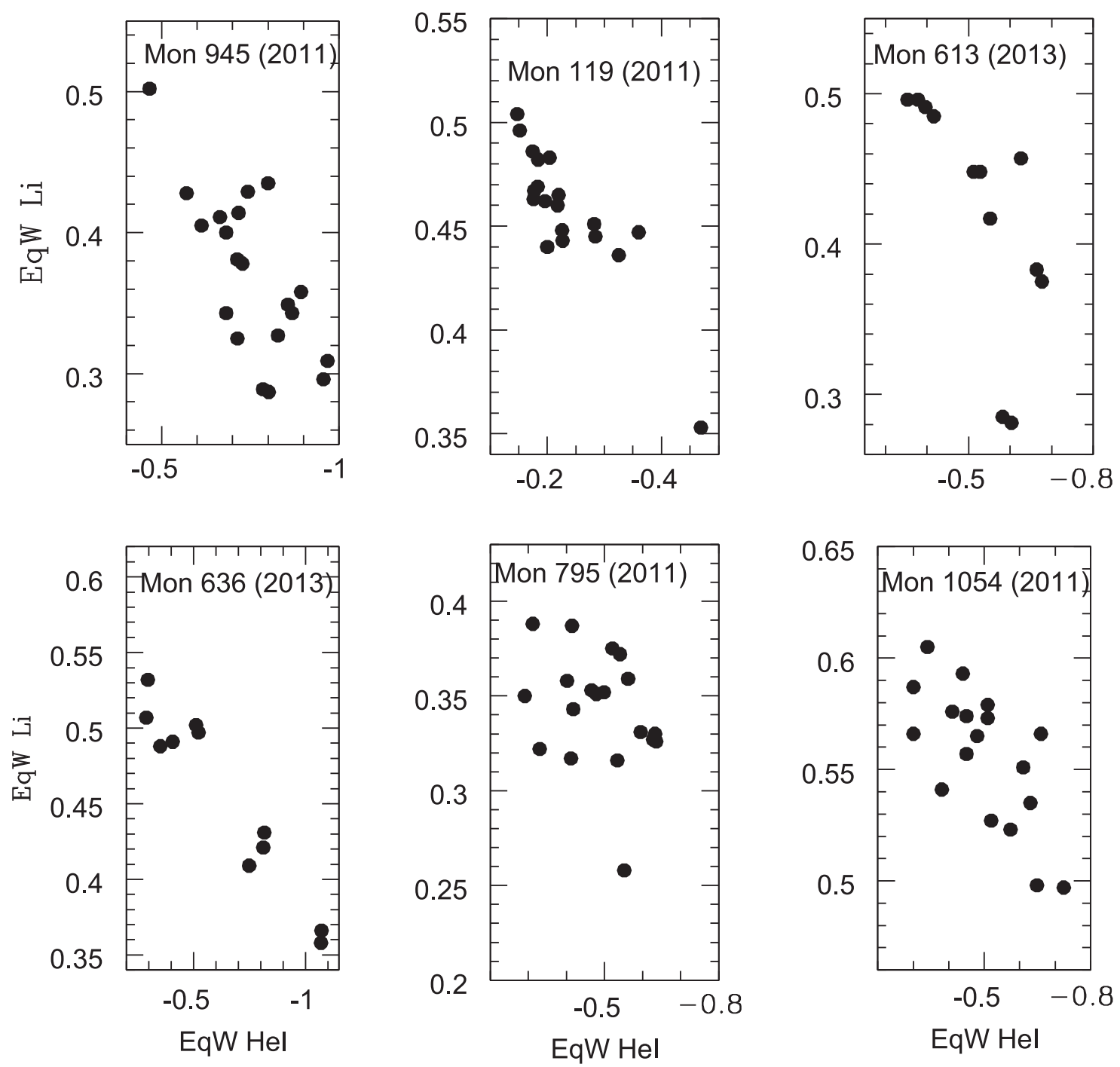

Figure 9. Correlation between $\mathrm{Li}$ I 6708 equivalent width and $\mathrm{He}$ I 6678 equivalent width for one star with an accretion-burst dominated light curve (Mon-945) and the five stars with stochastic light curves for which the He I line is significantly in emission for at least some of the spectra. The generally good correlation implies that the continuum variations observed for these stars are primarily due to variations in the accretion rate. The equivalent width for He I 6678 has been corrected for the influence of an $\mathrm{Fe}$ I absorption line at almost exactly the same wavelength by subtracting the expected equivalent width (as a function of spectral type) for the Fe I line from the measured $\mathrm{He}$ I equivalent width—see Appendix A.2 for more details.

with accretion burst dominated light curves), but similar to the average accretion rate for stars with variable extinction dominated light curves.

\subsection{Variable Accretion Inferred from Broadband Colors}

If the primary cause of the photometric variability of the stars in Table 2 is variable accretion onto the stellar photosphere, it should also be reflected in the broad-band photometric colors. When the accretion rate is high, the contribution to the optical flux from hot spots should be greatest, and the star should be both brighter and bluer. When the accretion rate is lowest, the hot spots should be at their weakest, and the star should be both fainter and redder.

As noted in Table 1, we have multi-epoch CFHT ugri photometry for NGC 2264 obtained about a month after the 2011 CoRoT/Spitzer campaign. Venuti et al. (2015) have described these data and used the data to quantify the level of accretion variability for the CTTS in NGC 2264. We now use the same photometry here to test whether accretion variability is the primary driver of the light curve morphology we have ascribed to the stochastic variables in the CoRoT data. As done by Venuti et al. (2015), for each CTTS, we have plotted the change in $r$ magnitude versus the change in $(u-r)$ color; where those data are well-correlated we have fit a straight-line to the distribution and derived the slope $(\Delta r / \Delta(u-r))$. Figure 11 shows a plot of these slopes for CTTS whose light curve morphologies we have ascribed to short-duration accretion bursts, to cold spots, and to our stars with stochastic light curves. The figure shows that the stochastic light curve stars have color slopes and amplitudes reasonably wellmatched to the short-duration accretion burst stars, and qualitatively different from stars whose light curves are dominated by cold spots. Stars whose light curves are dominated by variable extinction can have similar color slopes and amplitudes to those for the stochastic stars (Venuti et al. 2015), but the light curve morphologies of the two classes are quite different. 
Table 4

Other Quantitative Data Used in This Paper

\begin{tabular}{|c|c|c|c|c|c|c|}
\hline Mon-ID & $\langle\text { EqWLi }\rangle^{\mathrm{a}}$ & $\begin{array}{c}\text { Max-EqW-Li }{ }^{\text {b }} \\
(\AA)\end{array}$ & $Q$ & $M$ & $\begin{array}{c}v \sin i^{\mathrm{c}} \\
\left(\mathrm{km} \mathrm{s}^{-1}\right)\end{array}$ & $\begin{array}{c}v \sin i^{\mathrm{d}} \\
\left(\mathrm{km} \mathrm{s}^{-1}\right)\end{array}$ \\
\hline CSIMon-000119 & 0.48 & 0.52 & 0.62 & 0.05 & 21.0 & 24.8 \\
\hline CSIMon-000242 & $0.59^{*}$ & 0.53 & 1.18 & 0.32 & 14.9 & 18.5 \\
\hline CSIMon-000346 & $0.47^{*}$ & 0.53 & 0.95 & -0.08 & 21.5 & 18.2 \\
\hline CSIMon-000425 & $0.43^{*}$ & 0.50 & $\geqslant 1$ & 0.14 & 16.9 & 16.5 \\
\hline CSIMon-000457 & 0.25 & 0.33 & $\geqslant 1$ & -0.03 & 42.0 & 48.4 \\
\hline CSIMon-000491 & 0.32 & 0.46 & 0.99 & -0.12 & 72.0 & 67.9 \\
\hline CSIMon-000577 & $(0.42)$ & 0.42 & 0.70 & 0.09 & 35.0 & 34.5 \\
\hline CSIMon-000613 & 0.39 & 0.52 & 0.64 & -0.06 & 22.0 & 15.2 \\
\hline CSIMon-000636 & 0.44 & 0.60 & 0.65 & 0.04 & 25.0 & 24.9 \\
\hline CSIMon-000795 & 0.32 & 0.28 & 0.65 & 0.06 & 28.0 & 35.0 \\
\hline CSIMon-000985 & $0.47^{*}$ & 0.37 & 0.69 & 0.18 & 22.6 & 19.5 \\
\hline CSIMon-001054 & 0.53 & 0.62 & 0.71 & 0.18 & 20.0 & 20.1 \\
\hline CSIMon-001061 & 0.29 & 0.60 & 0.78 & -0.23 & $\ldots$ & 19.9 \\
\hline CSIMon-001234 & 0.43 & 0.52 & 0.61 & -0.03 & 24.0 & 15.9 \\
\hline CSIMon-001267 & $\ldots$ & $\ldots$ & $\geqslant 1$ & -0.06 & $\ldots$ & $\ldots$ \\
\hline CSIMon-006491 & $0.50^{*}$ & 0.48 & 0.87 & -0.01 & 17.0 & 15.8 \\
\hline CSIMon-006930 & $\cdots$ & $\ldots$ & 0.63 & 0.08 & $\ldots$ & $\ldots$ \\
\hline
\end{tabular}

Notes.

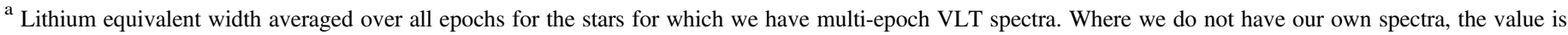
from measurement of the ESO-Gaia spectrum for this star, and these values are marked with an asterisk.

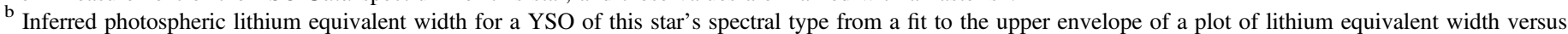
spectral type for our NGC 2264 stars for which we have VLT spectra.

${ }^{\mathrm{c}} v \sin i$ determined by comparing synthesized, rotationally spun-up model spectra to VLT/FLAMES observed spectra, as described in McGinnis et al. (2015).

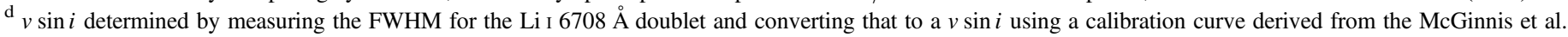
values. Where we have multi-epoch spectra, we measured FWHM for all the spectra and took the mean FWHM as the input.

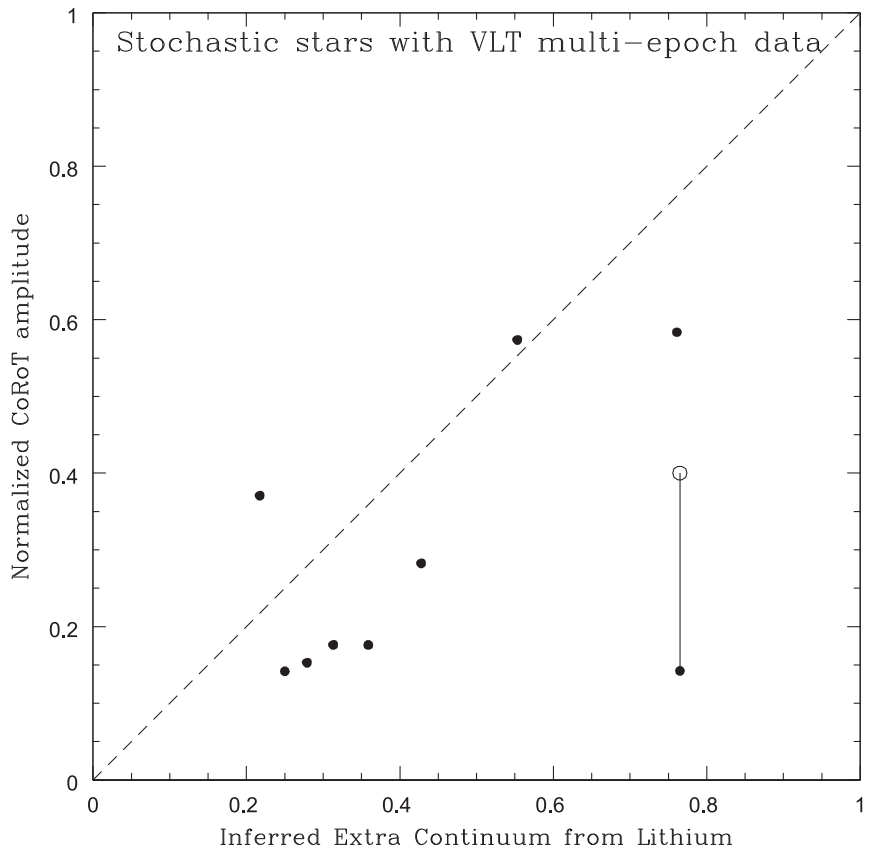

Figure 10. Comparison between the inferred range in the continuum flux at $6700 \AA$ A derived from our multi-epoch VLT spectra and the observed light curve amplitudes from our CoRoT data. The $y$-axis is the amplitude (in count rate) of the CoRoT light curve divided by the mean count rate. The dashed line indicates a one-to-one correlation. The open circle marks the light curve amplitude derived from our USNO data for a star that we believe had low variability during the CoRoT campaign but became significantly more variable during the following month, as indicated by our USNO and VLT data. We interpret the data shown here to be consistent with most of the optical variability for the stochastic stars as being a result of variable accretion onto their photospheres.

\section{ADDITIONAL CHARACTERIZATION OF THE STOCHASTIC LIGHT CURVE CLASS}

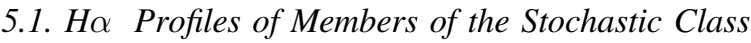

The shapes of emission line profiles for CTTS at least potentially provide clues to their accretion process (Lima et al. 2010; Kurosawa \& Romanova 2013). In Stauffer et al. (2014), we showed that NGC 2264 CTTS with accretion-burst light curves had $\mathrm{H} \alpha$ profiles that were distinctly different, on average, from those for the variable extinction light curve class. Specifically, the $\mathrm{H} \alpha$ profiles of the accretion burst stars are much more often centrally peaked and lacking in distinct absorption features compared to the variable extinction stars, while the variable extinction stars much more frequently show $\mathrm{H} \alpha$ absorption dips on the red side of the emission profile. These characteristics are explicable within the theoretical models (Kurosawa \& Romanova 2013), with the centrally peaked profiles being a prediction for stars with the highest accretion rates and the redward displaced absorption dips being a consequence of our line of sight intersecting warm gas that is accreting onto the stellar photosphere.

We have good multi-epoch VLT spectra with well-defined $\mathrm{H} \alpha$ profiles for eight of the stars in Table 2; for another five stars, we have good single epoch $\mathrm{H} \alpha$ profiles.

Figure 24, in the Appendix, shows typical $\mathrm{H} \alpha$ profiles for twelve of these stars (the $\mathrm{H} \alpha$ profile of Mon-613 is not shown in Figure 24, its $\mathrm{H} \alpha$ profiles are instead shown in Figure 12). $\mathrm{H} \alpha$ profiles for stars with accretion-burst dominated light curves and for stars whose light curves are dominated by variable extinction events can be found in Figures 6 and 26 of Stauffer et al. (2014). 


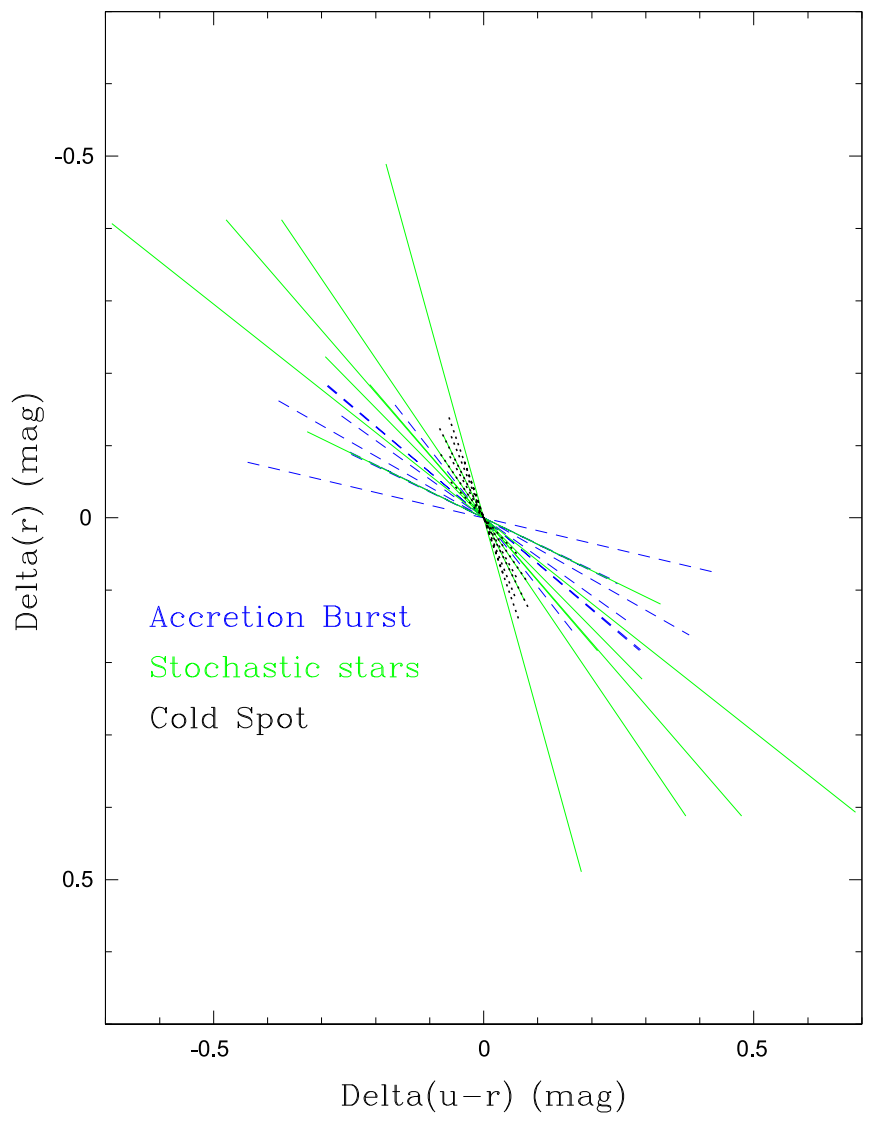

Figure 11. Comparison of the color-slopes $(\Delta r / \Delta(u-r))$ derived from multi-epoch CFHT photometry of NGC 2264 for stars whose CoRoT light curves have stochastic light curves to those that are either dominated by shortduration accretion bursts or by cold photospheric spots. The stochastic stars show very similar slopes to the accretion burst dominated sample, whereas the stars with cold spots have systematically higher slopes (because the spots are very red, leading to little change in $(u-r)$ color for a given change in $r$ magnitude). We have plotted the data for the largest amplitude cold spot light curves in order to be able to derive as accurate a slope as possible. Most stars with cold-spot light curves have significantly smaller amplitudes.

The most common feature of the $\mathrm{H} \alpha$ profiles of the stochastic stars is the presence of a blueshifted absorption dip. Six of the eight stars with multi-epoch data, and four of the five stars with single-epoch spectra have blueshifted absorption dips. For the stars with multi-epoch data, five of the six stars (Mon-119, Mon-457, Mon-491, Mon-613 and Mon-795) with blueshifted absorption dips have them for every spectral epoch - as is illustrated in Figure 12 for Mon-457 and Mon-613. The one star with intermittent blueshifted absorption dips is Mon1234, where only five of twelve spectra show blueshifted absorption dips (and eight of twelve epochs show redshifted absorption dips). For the stars in the accretion burst and variable extinction light curve classes for which we have multiepoch VLT spectra, about a third have persistent blue absorption dips; the number for the variable extinction stars is difficult to state accurately because their $\mathrm{H} \alpha$ profiles are often very complex and variable.

The spectra used for Figure 12 were obtained during a thirteen day interval in 2013, with two spectra obtained on nights $1,8,9,10$ and 13, and single spectra on nights 4 and 7 . The $\mathrm{H} \alpha$ profiles for Mon-457 were relatively stable over this period, with the primary variation being in the height of the blue peak, which first increased by about $20 \%$ and then decreased by about $50 \%$. The velocity offset of the blueshifted absorption dip was essentially constant (at about $-50 \mathrm{~km} \mathrm{~s}^{-1}$ ) for the entire two week interval. The $\mathrm{H} \alpha$ profiles of Mon-613 show greater variability, particularly in the height of the blue peak (which varies by more than a factor of two) and the velocity offset of the blue-absorption dip (which was generally about $-60 \mathrm{~km} \mathrm{~s}^{-1}$, but was $-100 \mathrm{~km} \mathrm{~s}^{-1}$ in the first spectrum). The last two spectra of Mon-613 show two blue-shifted absorption dips, one with $\Delta v=-165 \mathrm{~km} \mathrm{~s}^{-1}$ and the other with $\Delta v \sim-50 \mathrm{~km} \mathrm{~s}^{-1}$.

Similar but independent models of the $\mathrm{H} \alpha$ profiles for CTTS combining both magnetospheric accretion and disk winds have been created by Kurosawa et al. (2006) and Lima et al. (2010). Blue-shifted absorption dips of the type shown in Figures 12 and 24 are predicted to be present in the $\mathrm{H} \alpha$ profiles of CTTS primarily having moderately high mass accretion rates and generally having intermediate to low inclination angles $\left(i \lesssim 55^{\circ}\right)$. Such blue-shifted absorption dips are believed to arise from gas in a disk wind. If this is indeed the case, the fact that (when we have appropriate spectra) the blue absorption dips are fairly stable on timescales of two weeks or more indicates that the disk wind structure is also relatively stable on those timescales (longer than, for example, the expected photospheric rotation periods of these stars). A more detailed discussion of the $\mathrm{H} \alpha$ profiles of all the CTTS for which we have FLAMES data can be found in Sousa et al. (2015).

\subsection{Spectral Type and Light Curve Class}

It is reasonable to believe that light curve morphology could depend on the mass of the YSO. Higher mass YSOs are likely to have larger mean rotational velocities based on the Kraft "law" $\left(J / M \propto M^{2 / 3}\right.$; Kraft 1970) and hence perhaps might have shorter variability timescales. Higher mass YSOs are believed to often have more complex and more non-axisymmetric magnetic fields, while lower mass (but still $>0.5 M_{\odot}$ ) YSOs are expected to have more axisymmetric magnetic fields with strong dipolar components (Gregory et al. 2012). These characteristics are likely to affect the interaction between the star and its circumstellar disk and the resulting accretion patterns.

We do not have any direct mass indicator for the NGC 2264 members, but spectral type should provide a reasonable proxy for mass. Spectral type estimates are available for most of the NGC 2264 CTTS either from the published literature or our own spectra, or estimates of spectral types derived from multiband photometry (Venuti et al. 2014). Figure 13 compares the spectral type distributions for four sets of CTTS in NGC 2264: (a) the stochastic light curve class; (b) stars with AA Tau-type light curves or with strong, aperiodic flux dips due to variable extinction (McGinnis et al. 2015); (c) stars with accretion burst dominated light curves (Stauffer et al. 2014); and (d) stars with short-duration, nearly Gaussian-shaped flux dips (possibly due to dust entrained in accretion columns; Stauffer et al. 2015). Table 5, in the Appendix, provides the list of stars we have assigned to each group and our adopted spectral types.

The stars in all four light curve classes have a fairly wide range of spectral types. However, there are significant differences in the spectral type distributions, with the stochastic class having the highest fraction of early spectral type stars (four of 15 stochastic stars with estimated spectral types are spectral type $G$, versus only two of sixty for the other three classes combined), while the short-duration flux dip class has a 

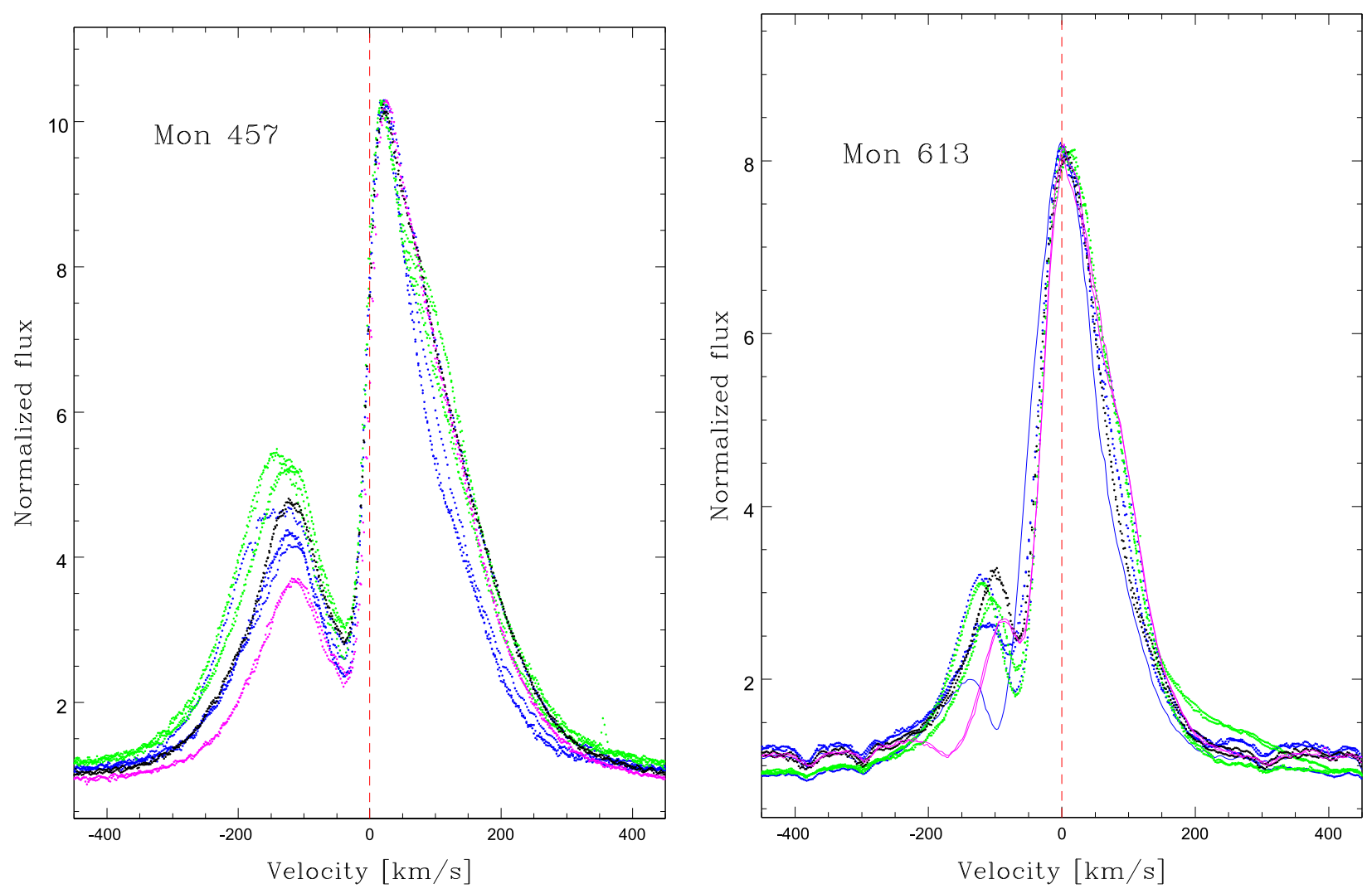

Figure 12. (a) $\mathrm{H} \alpha$ profiles for all twelve epochs obtained during the 2013 VLT/FLAMES campaign for Mon-457. The continuum has been normalized to 1.0 in all spectra, and the $y$-axis maximum scale has been adjusted so that the peak normalized flux at each epoch approximately coincides. The dot colors correspond to the observing epochs-the spectra from days 1, 4 and 7 are colored blue, spectra from days 8 and 9 are shown in green, day 10 in black, and day 13 in magenta. (b) Same, except for Mon-613.

larger fraction of $\mathrm{M}$ dwarfs. Simply assigning numerical equivalents to the spectral types $(\mathrm{G} 5=45 ; \mathrm{K} 7=57$; $\mathrm{M} 2=62$ ) and then taking averages, the mean spectral types for the four groups are K3 (stochastic), K5 (AA Tau), K7 (burst) and M0 (short-duration flux dip).

\subsection{Inferences on the Inclination of the Rotation Axis relative to our Line of Sight}

Knowledge of, or at least constraints on, the inclination of a young star's rotation axis to our line of sight can be very helpful in attempting to determine a physical mechanism to explain the observed variability. For the variable extinction light curve class, if one assumes that the extinction events occur when structures in the inner disk pass through our line of sight, then the presence of the flux dips plus a theoretically modeled disk scale height constrain the inclination of the disk to be $\gtrsim 60^{\circ} .{ }^{21}$ For stars with stable, periodic light curves due to non-axisymmetrically distributed spots, the shape of the light

\footnotetext{
${ }^{21}$ Following the simple but physically well-justified CTTS disk model described in Bertout (2000), we assume that on average, there is some inclination angle $i_{\text {thick }}$ greater than which the star is always obscured, there is a range of inclinations between $i_{\text {thick }}$ and $i_{\text {thin }}$ where variable extinction events may occur, and for inclinations less than $i_{\text {thin }}$ no disk-related extinction events occur. From the census of CTTS light curves in NGC 2264 (Alencar et al. 2010; McGinnis et al. 2015; Stauffer et al. 2015) we set the fraction of optically visible CTTS with variable extinction events at $25 \%$. Because the estimated line of sight inclination to the disk for the prototype CTTS AA Tau is $i=70^{\circ}-75^{\circ}$ (Bouvier et al. 2013), we adopt $i_{\text {thick }}=75^{\circ}$. With those two parameters set, the Bertout (2000) model then yields $i_{\text {thin }} \simeq 60^{\circ}$.
}

curve and the presence or absence of eclipses place constraints on the latitude of the spot(s) and our view angle to the star. We can draw some inferences on the disk inclinations for the stochastic light curve class members simply from their light curve morphology; we can infer a little more from their spectra - as we describe below.

Members of the stochastic light curve class are in all cases CTTS. They have circumstellar disks that extend inward close enough to the star's photosphere to produce class II SEDs. By definition of our light curve classes, a star in the stochastic class does not have deep flux dips due to variable extinction events, and therefore the inclination of our line of sight to their disks is almost certainly $\lesssim 60^{\circ}$. As noted in Stauffer et al. (2015), two of the stochastic light curve stars do have probable short-duration flux dips in their light curves (one dip each in 2008 and 2011 for Mon-119, and two dips in 2011 for Mon-577-see Figure 2). These short duration flux dips may arise from dust in accretion columns-and hence from dust above the expected disk upper surface layer. Even if the occulting dust is nominally part of the disk material, the short duration and shallowness of these flux dips suggest that the dust would be located in the upper surface layer of the disk. Hence, even for these stars, we expect $i \lesssim 60^{\circ}$.

If there were well-measured periods, $v \sin i$ 's, and radii available for the stochastic stars, it would be possible to directly estimate $\sin i$. However, periods are not known (more or less "by definition") for the stochastic stars. Nevertheless, one might at least determine if the stochastic stars have an unusual $v \sin i$ distribution compared to other CTTS in NGC 2264, and 


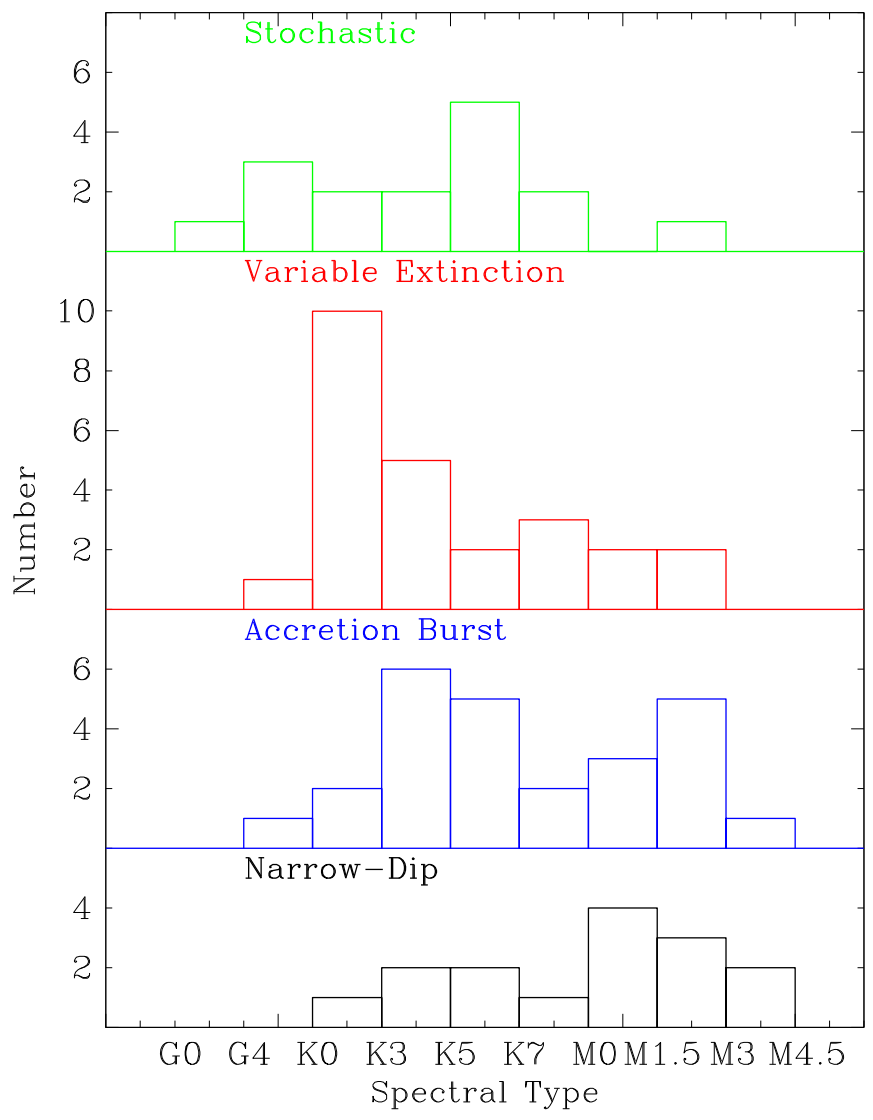

Figure 13. Histogram of the spectral type distribution for the stochastic light curve class members compared to histograms for three other CTTS light curve classes. See discussion in Section 5.2.

from that either be able to draw inferences about their inclinations or their intrinsic rotational velocities ${ }^{22}$. Even that exercise is limited due to the spectral types of the stochastic stars (Figure 13), because the stochastic stars include an excess (relative to the other light curve classes) of relatively early type stars $(\leqslant \mathrm{K} 3)$. These early type stars will arrive on the ZAMS as $\mathrm{A}$ or $\mathrm{F}$ stars, and hence even on the PMS will have systematically higher rotational velocities than lower mass CTTS. The actual $v \sin i$ 's, as illustrated in Figure 14, confirm this bias, with many of the most rapidly rotating stars in all three plotted CTTS groups having spectral type K3 or earlier. Excluding the early spectral types, the distribution of $v \sin i$ 's for the three CTTS groups looks quite similar. The average $v \sin i$ 's confirm this: the mean $v \sin i$ 's for the three groups are $19.0 \mathrm{~km} \mathrm{~s}^{-1}(n=10), 20.8 \mathrm{~km} \mathrm{~s}^{-1}(n=17)$, and $18.7 \mathrm{~km} \mathrm{~s}^{-1}$ $(n=15)$ for the stochastic, accretion burst, and variable extinction classes, respectively. The accretion burst mean is significantly influenced by one very discrepant star-Mon-7, with $v \sin i=85 \mathrm{~km} \mathrm{~s}^{-1}$; excluding Mon-7, the accretion burst class average $v \sin i$ becomes $16.9 \mathrm{~km} \mathrm{~s}^{-1}$.

The essential equality of the mean $v \sin i$ 's for the stochastic and variable extinction stars is perhaps unexpected. Given our conclusions concerning the line of sight inclination to the rotation axes of the two classes $\left(i \gtrsim 60^{\circ}\right.$ for the variable extinction class and $i \lesssim 60^{\circ}$ for the stochastic class), and assuming a random distribution of inclinations within those

\footnotetext{
22 A brief description of our process for estimating $v \sin i$ 's is provided in the Appendix.
}

ranges, one would predict $\langle\sin i\rangle_{\mathrm{var}-\mathrm{ext}} /\langle\sin i\rangle_{\text {stoch }} \sim 1.5$; hence for $\langle v \sin i\rangle=18 \mathrm{~km} \mathrm{~s}^{-1}$ for the variable extinction class, one would have expected $\langle v \sin i\rangle=12 \mathrm{~km} \mathrm{~s}^{-1}$ for the stochastic class. At face value, therefore, the equality of their average $v \sin i$ 's suggests that the stochastic stars might be comparatively rapid rotators. However, because the mean value for the stochastic class was derived from only ten stars, we believe it prudent to simply conclude that it is unlikely that the stochastic stars are unusually slowly rotating.

\subsection{Variability Timescales}

One can obtain useful constraints on the timescale associated with accretion variability in the stochastic stars from our multiepoch VLT/FLAMES spectra. If the accretion rate changed only slowly with time (e.g., with a several day time constant), then spectra taken on the same night, or on successive nights, would have lithium equivalent widths or He I $6678 \AA$ fluxes that differ from each other by significantly less than spectra separated by several days. We have seven stars with multiepoch VLT data (from either 2011 or 2013) where we can make this test using lithium, and four stars where this test is possible for the helium emission line (the other stars either never or rarely show helium emission). For each star, we identified time lags with at least three pairs of epochs with that time lag (often there were more than three). For each time lag, we calculated the mean absolute deviation between the measured equivalent widths for each pair of epochs. Figure 15 shows the time lag plots for lithium $6708 \AA$ and helium $6678 \AA$. As an ensemble, the plotted data indicate that most of the variability occurs on timescales of order a day or less.

Photometric light curves, of course, provide a much more direct means with which to derive variability timescales. For stars with periodic light curves, one can easily determine that period and often associate that period with a physical mechanism (e.g., the rotation period of the star). For stars with aperiodic light curves, it is much more difficult to derive a timescale which correlates well with the shape one "sees" in the light curve and which can be convincingly linked to a physical mechanism. After some experimentation, we decided to derive timescale estimates based on an algorithm that measures how long it takes for the light curve to change by a given fraction of its full amplitude. Specifically, for every point in the light curve, the algorithm looks both forward and backward in time, and determines the time lag needed for the absolute difference in magnitude to change by a given amount. We then average the derived time lag for every light curve point to determine the mean time lag for that delta magnitude. We calculate those timescales for steps in delta magnitude ranging from 0.005 mag up to one half the full magnitude range in the light curve. When portions of the light curve seem to have linear trends in magnitude (only the first half of the Mon-425 light curve, and the last third of the Mon-795 light curve-see Figure 2 and Figure 3), we first detrend the light curve to remove that linear trend. We associate a characteristic time for the star as the derived mean time lag for a delta magnitude corresponding to half the full amplitude of the light curve $\mathrm{e}^{23}$. These characteristic timescales are provided in Table 2. The timescales range from half a day to of order five days, with most of the stars having

\footnotetext{
23 This algorithm returns timescales that are quite similar to what would be derived from a peak-finding program, for peaks larger than half the full amplitude, but avoids the difficulty of determining when a peak occurs.
} 
timescales near one day-in accord with the analysis of the VLT/FLAMES multi-epoch data.

In principle, the way in which the mean time lag depends on delta magnitude should encode information on the light curve shape. Figure 16 plots this relation both for our stochastic stars and for a comparison set of stars with periodic light curves. The plot shows nearly linear correlations for the periodic light curve stars, with all of the stochastic stars falling below that relationship. The stochastic stars with short characteristic timescales ( $\tau<1.2$ days) in general fall closer to the periodic relation than the stochastic stars with longer characteristic timescales.

At a macroscopic level, it is reasonable to think of the light curves for the stochastic variables as reflective simply of a global accretion process whose $d M / d t$ varies in a seemingly random way. Such light curves can be well-matched by simulations with a damped random walk process (e.g., Findeisen et al. 2015). However, on a microscopic level, it is possible that all of the variability arises from individual accretion events creating transient hot spots of variable size, temperature and lifetime. If so, the properties of the observed light curves could be used to place constraints on the properties of the individual accretion events. We have created a simple Monte Carlo process model to explore this idea.

Our model produces light curves of total duration 34 days, from which we extract the central 30 day portions (to minimize edge effects). We distribute $N$ Gaussian flux bursts during the observing window, with each burst having a height, width and central epoch chosen randomly within specified bounds from a uniform distribution. We also add a continuum level to simulate the contribution to the light from the stellar photosphere. We vary the number of bursts and the parameter bounds (a) to match the observed light curve amplitudes; (b) to match the mean veiling (the fraction of the light from accretion compared to that from the photosphere); (c) to yield values of $Q$ and $M$ appropriate for stars with stochastic light curves; and (d) to yield timescales as judged from our $\Delta m$ versus $\Delta t$ algorithm that approximately match specific stochastic class stars.

Figure 17(a) shows a simulated light curve ("Case A") whose morphology, amplitude, mean veiling and timescale ( $\tau=1.4$ days) was designed as a reasonable match to the CoRoT light curve of either Mon-346 or Mon-577 (see Figure 2). The parameters for this model were $N$ (number of bursts in 30 days $)=300$, FWHM $=0.2-0.7$ days; height $=$ 5.0-20.0 (in "counts"), and photospheric continuum level = 500 (in "counts"). $M$ is 0.14 for this light curve, and the $Q$ statistic indicates the light curve is random-hence $Q$ is set arbitrarily to 1 .

Figure 17(b) shows a simulated light curve ("Case B") whose morphology, amplitude, veiling and timescale ( $\tau=3.3$ days) was designed to reasonably match the CoRoT light curve for Mon-457 (see Figure 2). The parameters for this model were $N=300, \quad$ FWHM $=0.9-2.7$ days, height $=2.0-10.0$, and photospheric continuum level $=400 . M$ is -0.19 for this light curve, and the $Q$ statistic indicates the light curve is randomhence $Q$ is set arbitrarily to 1 .

Figure 17(c) shows that as the frequency of bursts decreases, individual bursts become more visible and the light curve evolves to better resemble our accretion burst light curve class (Stauffer et al. 2014). The parameters for this light curve were
$N=30, \quad$ FWHM $=0.5-1.5$ days,$\quad$ height $=5.0-18.0, \quad$ and photospheric continuum $=400$.

Finally, Figure $17(\mathrm{~d})$ provides a $\Delta t$ versus $\Delta m$ plot for the Case A and Case B models. Also plotted are the $\Delta t, \Delta m$ values for two idealized periodic light curves - a sine wave and a sawtooth function. The models designed to look like our stochastic stars have $\Delta t, \Delta m$ loci in the diagram that mimics the loci for the stochastic stars (Figure 16).

We view this exercise as primarily a proof of concept. The stochastic light curves can indeed be simulated with many small, discrete flux bursts. There must be many bursts, on average, per day in order to yield light curve shapes that appear symmetric about their median value. However, the durations of the events must be kept short in order that the summed flux from the accretion events does not become too large and the light curves do not become too smooth (low amplitude). The parameters we have chosen for Case A and B are presumably not unique, but they at least provide possible choices that could yield light curves similar to those we observe in NGC 2264.

\subsection{Correlation of Optical and IR Light Curves}

The optical photons from CTTS come primarily from the photosphere and the hot spots produced by accretion shocks. The IRAC (3.6 and $4.5 \mu \mathrm{m})$ photons also originate from these regions, as well as from warm dust in the inner circumstellar disk. For stars with only small IR excesses from warm dust, and where there is little or no variable extinction, the optical and IR light curves should be well-correlated (but possibly with significantly different amplitudes), regardless of the physical mechanism driving the variability. For stars where the disk photons dominate at IRAC wavelengths, the optical and IR light curves could be quite uncorrelated, depending on the physical mechanisms involved. The most likely physical processes driving variability at IRAC wavelengths are: (a) heating of the dust in the inner disk by reprocessing of the photons emitted from the star's surface; (b) changes in the inner disk dust surface area exposed to the stellar photon bath (e.g., from instabilities lifting more dust into the disk photosphere); and (c) viscous, self-heating of the inner disk. The optical-IR correlation can also be affected by details of the geometry-a transient hot spot on the side of the star facing us produces a flux burst most evident in the optical light curve, but if that hot spot is not located in a position where it is seen by the warm dust that dominates the IRAC light, then it may have only a minor impact on the IRAC light curve. Conversely, a transient flux burst on the back side of the star would have no impact on the optical light curve that we see, but could have a significant impact on the IR light curve if the IR flux is dominated by photons from warm dust in the inner disk wall that faces the back side of the star, as is in fact likely. As discussed extensively in Whitney et al. (2013), the correlation between the optical and IR light curves of the stochastic stars therefore can provide important clues to the physics underlying their variability; we discuss that correlation for our stochastic stars now.

Table 2 provides two quantities related to the optical/IR variability issue - the estimated disk to photosphere flux ratio at $4.5 \mu \mathrm{m}$ (column 6), derived from our single-epoch photometry obtained prior to 2010, and an optical-to-IR Stetson index (column 8), derived from our 2011 CSI 2264 campaign data. The disk to photosphere ratio ranges from 0.4 for Mon242 , a star with very little IR excess, to $>10$ for Mon-985, a 


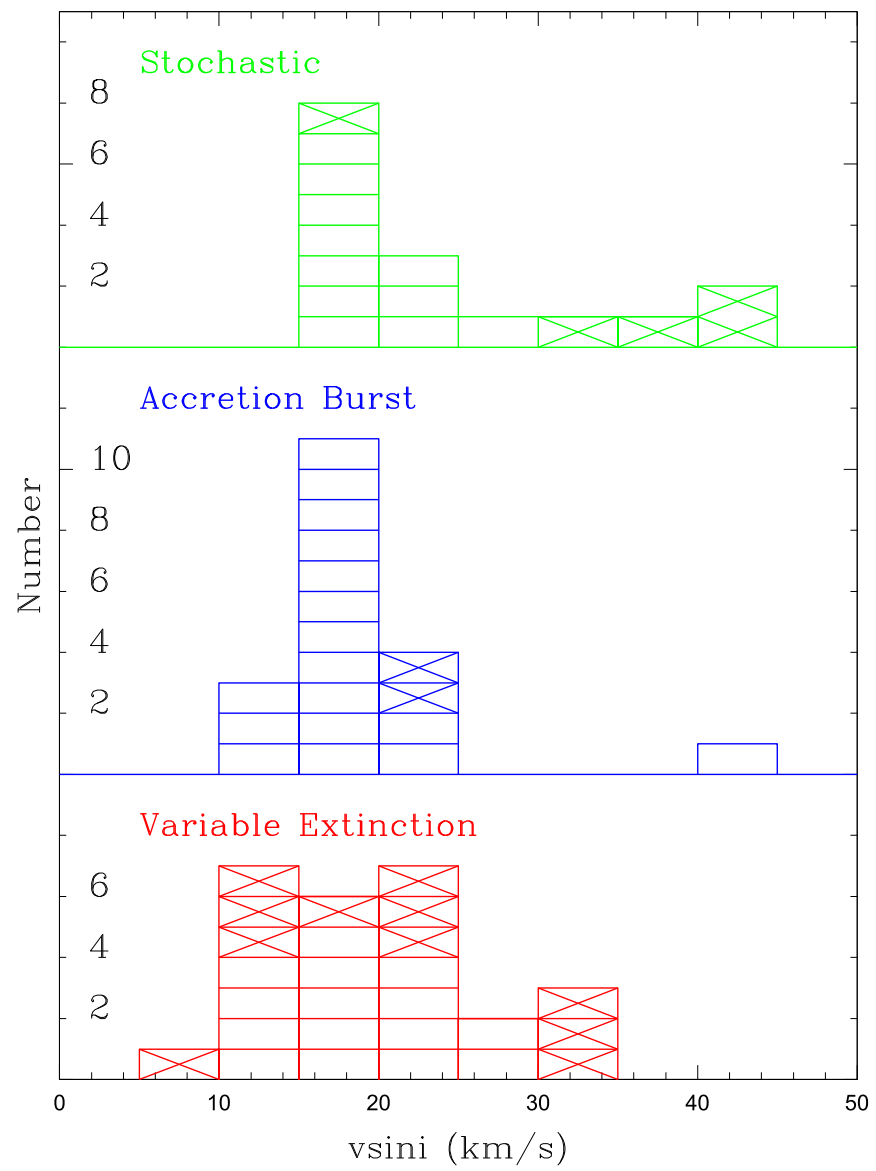

Figure 14. Histogram of estimates of the projected rotational velocity derived from Gaussian fits to the $\mathrm{Li}$ I $6708 \AA$ doublet for the variable extinction, accretion burst, and stochastic light curve classes. Stars with spectral type K3 or earlier are marked with a cross; not unexpectedly, these stars include most of the rapidly rotating CTTS in the three classes.

star with a very large IR excess. The Stetson index (see Cody et al. 2014 for a more thorough discussion) ranges from 0.41 (largely uncorrelated) to $>2$ (well-correlated). Figure 18 shows an overlay of the CoRoT and IRAC light curves for four of the stochastic stars where the Stetson index indicates a good degree of correlation between the optical and IR light curves.

The stars in Figure 18 are arranged in order (top-left to bottom-right) of increasing disk dominance at $4.5 \mu \mathrm{m}$, with disk-to-star flux ratios of $0.4,2.9,5.4$, and $>10$ for Mon-242, 119,636 , and 985, respectively. Visual examination of the four panels indicates that they are also arranged approximately in order of the degree to which the amplitude and shape of the short-timescale IR fluctuations match those in the optical. The optical and IR light curves for Mon-242 track each other very well on short timescales in both shape and amplitude. Mon-119 generally shows well-defined IR peaks at location of the optical peaks, with amplitudes often of order half that as shown in the optical. Mon-636 and Mon-985, both having disk-dominated IRAC photometry, show much lower amplitude variability in the IR compared to the optical, but often appear to have barely discernible features at the location of structures in the optical light curve. A better view of the optical-IR correlation for these two stars is provided in Figure 19, where the IRAC light curves are shown magnified by a factor of 3 in the $y$-axis. This plot shows that, in fact, there is still a good correlation in the optical and IR short-timescale morphology, but the amplitudes of the
IRAC peaks are on average about one third the amplitude of the optical peaks.

Close examination of the four panels of Figure 18 shows some optical features which appear to be completely lacking in IR counterparts. Good examples are flux excesses in the optical light curve for Mon-119 at days $\sim 12$ and $\sim 20$, with no evidence at all for a feature at those times in the IRAC light curve. There are no obvious inverse situations, where an isolated IRAC flux peak is lacking a simultaneous CoRoT peak.

Assume for the moment that the accretion hot spots have effective temperatures of order $8000 \mathrm{~K}$ and that our CTTS are all around spectral type K4. If the hot spot emitted as a blackbody, it would have $R-[3.6] \sim 0$, and the "quiescent" photosphere would have $R-[3.6] \sim 2$. In the absence of any warm dust, an accretion burst causing a 0.2 mag brightening in the optical light curve (comparable to the light curve amplitude for a typical stochastic star) would cause only a $\sim 0.03$ mag brightening at $4 \mu \mathrm{m}$. For stars with large IR excess, where the disk dominates the $4 \mu \mathrm{m}$ flux by a large factor, the flux variation at $4 \mu \mathrm{m}$ directly from the photosphere would be even lower. Because the emitted flux from accretion hot spots has a spectral shape closer to $\mathrm{F}_{\lambda} \sim$ constant rather than a blackbody spectrum (Hartigan et al. 1989; Basri \& Batalha 1990), this estimate is somewhat inaccurate, but we believe the basic conclusion is still valid. Therefore, it is unlikely that the flux variation at IRAC wavelengths seen in any of the stars in Figure 18 is directly a result of the variable photospheric contribution.

Assume now also that radiative heating dominates the energy budget for the warm dust responsible for the flux at IRAC wavelengths, and also assume that the system geometry is such that the transient hot spot is well positioned to be seen by us and by the warm dust in the inner disk. In that case, a change in the luminosity of the star of $\Delta m(\mathrm{bol})_{\text {star }}$ due to appearance of a transient hot spot should approximately result in a similar increase in the luminosity of the warm dust. A $8000 \mathrm{~K}$ hot spot should have an SED peaked near $4000 \AA$, close enough to the center of the broad CoRoT bandpass such that a $20 \%$ change in the CoRoT light curve flux should correspond approximately to a $20 \%$ change in luminosity of the hot spot. For $1000 \mathrm{~K}$ dust, $\lambda_{\max } \sim 4 \mu \mathrm{m}$, and a $20 \%$ change in the IRAC light curve should correspond approximately to a $20 \%$ change in the luminosity of the warm dust. This suggests that there could be a good correlation between optical and IR light curves for stars with disk-dominated fluxes at IRAC wavelengths, which we in fact do see (Figure 19). To be explained still are the variable, and sometimes small IR-to-optical amplitude ratios, and the optical peaks with no apparent IR counterpart.

It is possible to adjust the geometry to reduce the response of the disk to the hot spot luminosity variations by placing the hot spots at higher stellar latitude. This reduces the projected area of the hot spot as seen by the inner disk dust roughly as the cosine of the spot latitude, so that a spot at $70^{\circ}$ latitude would have about half the disk heating impact of a spot of the same physical size if at $45^{\circ}$ latitude. For an observer located near the rotation axis of the star, spots at such high latitudes would always be visible and the portion of the inner disk-rim they "light up" should have similar view angles to the observerwhich would result in a good correlation between the optical and IR peaks and a rarity of cases where there is an optical peak without an IR counterpart. As either the spot latitude decreases or the inclination of the observer's line of sight to the system 

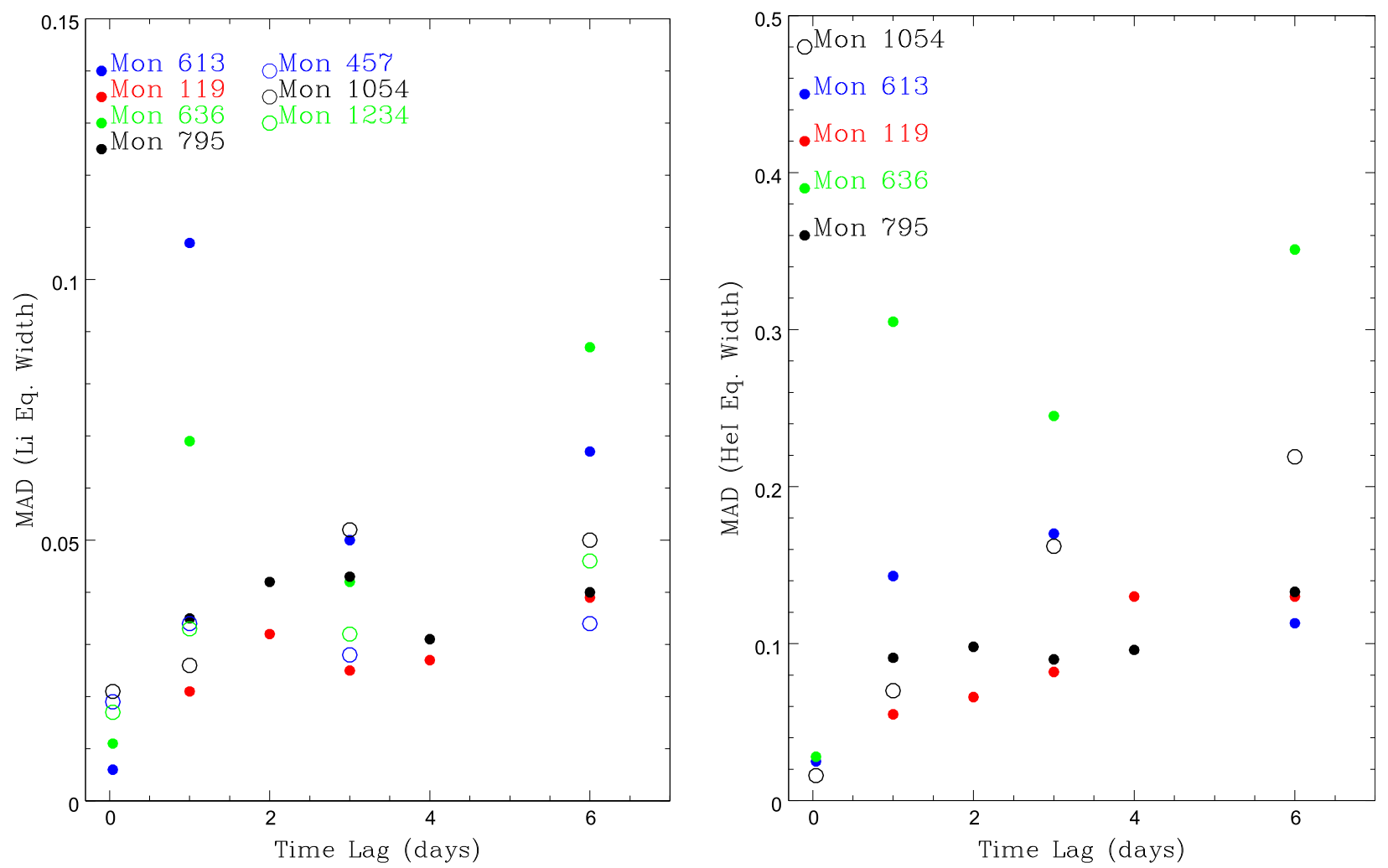

Figure 15. (a) Mean absolute difference for lithium equivalent widths measured at two different epochs as a function of the time lag between the two epochs. (b) same as for (a), except the feature measured is the narrow emission component for He I $6678 \AA$.

rotation axis increases, the chance for a transient hot spot occurring where it is not visible to the observer increases. Therefore, the observed correlation between the optical and IR light curves favors both relatively high spot latitudes and relatively low inclinations of the system rotation axis to our line of sight. This geometry also naturally reduces any periodic signature in our light curve due to the star's rotation.

\section{SUMMARY AND CONCLUSIONS}

Based on the preceding sections, we can summarize the properties that characterize stars whose optical light curves fall into our stochastic class as follows.

1. By selection, their optical light curves appear symmetric about their median level, with no preference for discrete downward flux dips (variable extinction) nor upward flux bursts (short-duration flux bursts). The lack of flux dips due to extinction excludes stars where the inclination of our line of sight to the circumstellar disk is large, implying $i \lesssim 60^{\circ}$.

2. By selection, their optical light curves show very little periodic signature.

3. The fraction of the CTTS in NGC 2264 having optical light curves of this type is about $10 \%$.

4. As judged by their UV excesses, $\mathrm{H} \alpha$ equivalent widths, and veiling of optical absorption lines, the stars with stochastic light curves have relatively low to moderate accretion rates. Their mean veiling level is generally of order $10 \%-20 \%$.

5. Their CoRoT light curves show amplitudes generally in the range of $10 \%-30 \%$.
6. In all cases, their broad-band IR photometry indicates SED slopes consistent with Class II.

7. The members of the stochastic light curve class have spectral types ranging from early $G$ to early $M$, with a somewhat earlier mean spectral type than our other CTTS light curve classes.

8. The stochastic stars have small reddening, as judged from the $J-H$ versus $H-K$ color-color diagram and from comparison of their observed optical colors to colors predicted based on their spectral types.

9. Their $\mathrm{H} \alpha$ profiles are most often characterized by blueshifted absorption dips that are usually present with similar shape and amplitude at all epochs, best explained as due to gas in a disk wind seen from moderate to low inclination angles.

10. They have veiling variability and color changes derived from multi-epoch photometry indicative of a variable blue continuum source, consistent with expectation for photospheric hot spots whose size or temperature change with time.

11. Variability timescales for the stochastic stars derived either from the light curves or from multi-epoch spectroscopy are generally of order 1-2 days.

12. There is usually a good correlation between the optical and IR light curve morphologies on short timescales, but often much lower amplitudes for the IR fluctuations; short timescale events at one wavelength but not the other are quite rare. These characteristics are best explained by placing the transient hot spots at high stellar latitudes and the observer at low inclination angles to the star system rotation axis. 


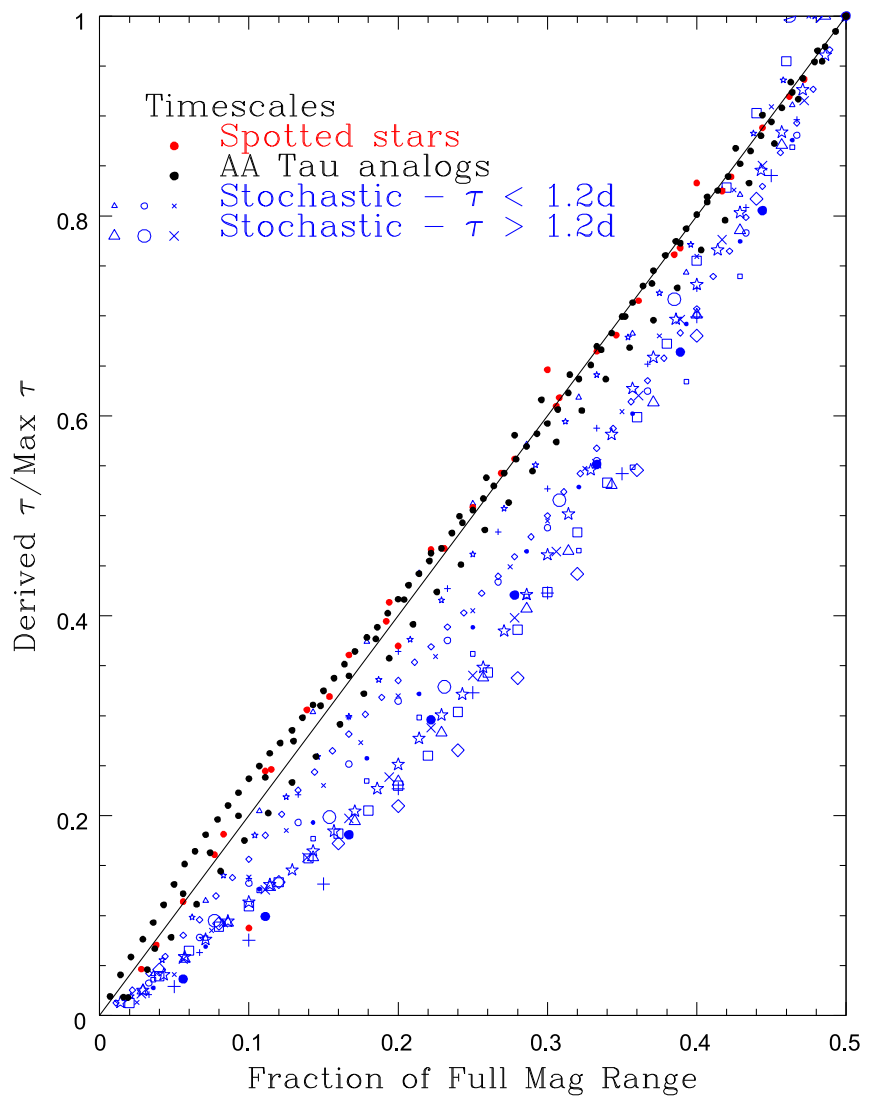

Figure 16. Correlation between the mean time lag for the light curve to change by a given $\Delta$ magnitude and $\Delta$ magnitude for the stochastic stars and for a sample of stars with periodic light curves including both AA Tau analogs and stars with cold spots. For each star, the $y$-axis has been scaled so that a value of 1.0 corresponds to the time lag for a $\Delta$ mag of half the full amplitude. Stars with cold-spot dominated light curves are shown as red dots, AA Tau analogs as black dots, stochastic stars with short timescales are shown as small blue symbols (a different symbol type for each star), and stochastic stars with long timescales are shown as large blue symbols.

Our data show that the light curve variability of the stochastic class arises from their having highly variable photospheric mass accretion. Of course, all CTTS presumably have variable mass accretion rates. For some CTTS, most notably the variable extinction light curve class, other processes produce fluctuations in the optical flux of much larger amplitude which mask the comparatively small changes in brightness due to the variable accretion rate. Where such camouflage is not present, there appears to be (at least) three light curve morphologies where accretion processes drive the variability. Stars with one or two dominant, stable accretion flows (funnel flow accretion) where the star's rotation is the dominant timescale can produce quasi-periodic, symmetric ("hot spot") light curves (Cody et al. 2014), like that shown in Figure 1(e). Stars with the highest accretion rates have light curves dominated by relatively strong, short-duration accretion bursts that are usually random in time but sometimes occur as periodic burst groups (Stauffer et al. 2014; Blinova et al. 2015). What is the physical driver for the variability shown by the stochastic light curve class?

We believe a clue to the answer to this question can be found by considering the members of the variable extinction class, and in particular one of the prototypes-AA Tau. Based on an extensive Zeeman Doppler imaging study of AA Tau, Donati et al. (2010) concluded that (a) its magnetic dipole axis is tilted by $\sim 20^{\circ}$ relative to its rotation axis; (b) the primary accretion hot spots are located near latitude $=70^{\circ}$; (c) its mean mass accretion rate to the stellar surface is quite small, with veiling levels ranging from near zero up to $\sim 0.2$; (d) that the accretion rate varies significantly on timescales less than the stellar rotation period. Concerning the variability in the inferred accretion rate, they also concluded that "... the accretion variability observed for AA Tau mostly relates to the variable efficiency at which the disc material succeeds at entering the closed magnetosphere in the propeller regime, rather than to an intrinsic variability of the accretion rate within the inner disc."

This characterization of accretion in AA Tau very nearly matches our description of accretion in the stochastic light curve class, and we believe this points to the most likely physical model for their variability. Our line of sight to the variable extinction class members must be $i \gtrsim 60^{\circ}$. There must be at least as many CTTS with the same accretion properties but seen at $i \lesssim 60^{\circ}$. Our data support identifying at least many of these low-inclination counterparts to the variable extinction class stars with members of the stochastic light curve class. Adopting the Donati et al. (2010) conclusion for AA Tau, we suggest that instability processes in the disk photosphere regulate the flow of material onto the magnetic field lines in the funnel flow, thereby also leading to the stochastically variable flux from hot spots on the stellar surface. Some fraction of the variable extinction stars may have more stable accretion and hence have hot spots that are relatively stable in size and location; when seen at moderate inclination, these stars should have light curves that are more or less periodic. Cody et al. (2014) labeled such stars as quasi-periodic, symmetric. Future synoptic studies of starforming regions should be conducted to determine whether stars with quasi-periodic, hot-spot light curves can transition to the stochastic light-curve morphology (and, if so, how the statistics for that transition compares to the statistics for the transition between AA Tau type light curves and the aperiodic flux dip light curve class).

This work is based on observations made with the Spitzer Space Telescope, which is operated by the Jet Propulsion Laboratory, California Institute of Technology, under a contract with NASA. Support for this work was provided by NASA through an award issued by JPL/Caltech. This research was carried out in part at the Jet Propulsion Laboratory, California Institute of Technology, under a contract with the National Aeronautics and Space Administration and with the support of the NASA Origins of Solar Systems program via grant 11-OSS11-0074. RG gratefully acknowledges funding support from NASA ADAP grants NNX11AD14G and NNX13AF08G and Caltech/JPL awards 1373081, 1424329, and 1440160 in support of Spitzer Space Telescope observing programs. SHPA, AS and PTM acknowledge support from CNPq, CAPES and Fapemig.

Facilities: Spitzer (IRAC), CoRoT, CFHT (MegaCam), VLT (FLAMES). 

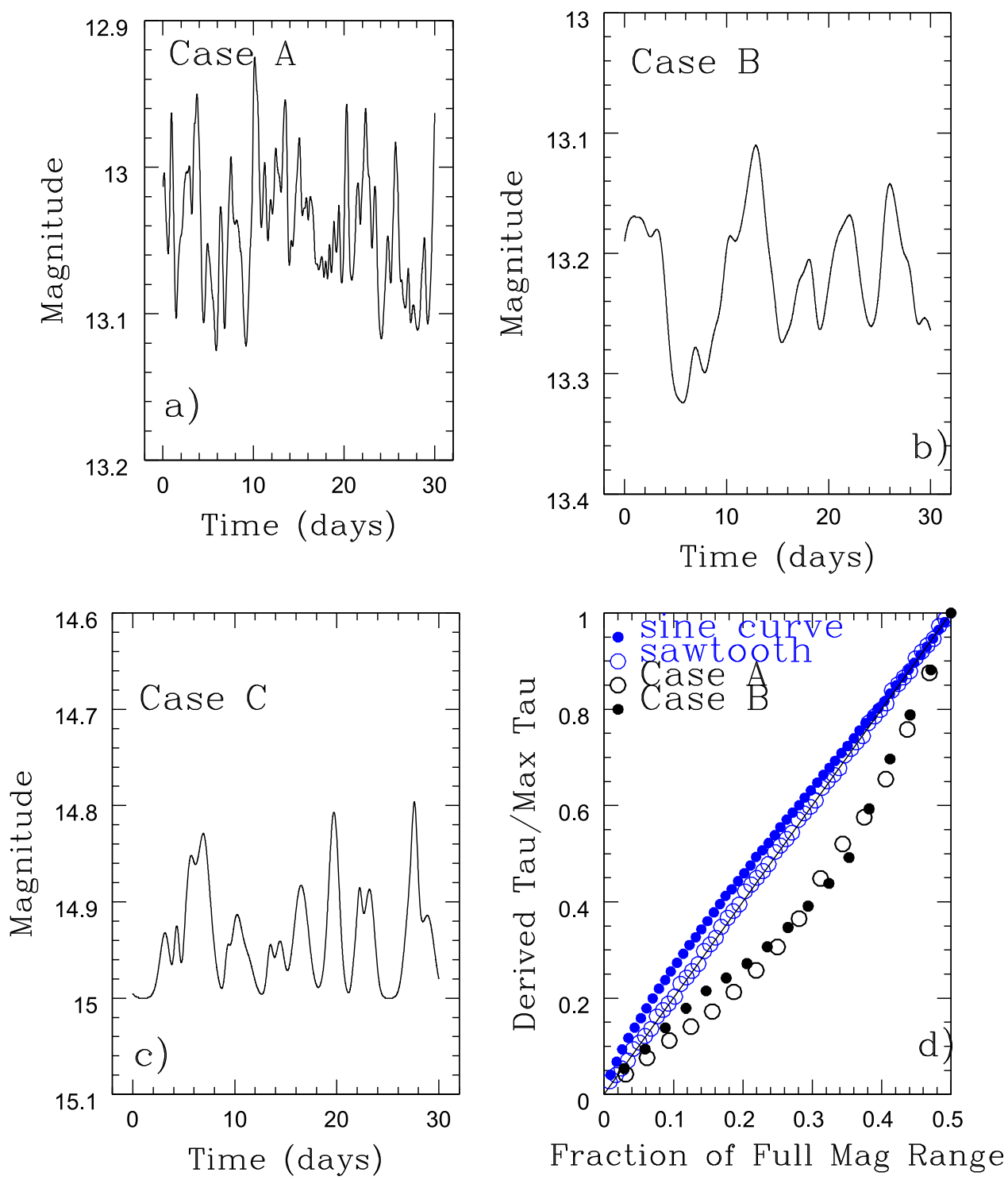

Figure 17. Results from Monte Carlo models aimed at simulating the light curve morphologies of the stochastic light curve class. See discussion in Section A.3. The photospheric continuum level in plots (a)-(c) are 13.25, 13.49 and 15.0 mag, respectively. (a) Simulated light curve intended to emulate the morphology of a short timescale stochastic star (e.g., Mon-346 or Mon-577); (b) Simulated light curve intended to emulate the morphology for a long timescale stochastic star (e.g., Mon457); (c) Simulated light curve, intended to emulate a light curve we would classify as accretion-burst dominated (Stauffer et al. 2014); (d) $\Delta t$ vs. $\Delta m$ plot for the light curves in panels (a) and (b), compared to two idealized light curve types (sine wave and sawtooth).

\section{APPENDIX}

\section{A.1. CoRoT Light Curves for Additional Stars Whose QM Metrics Match that for Stars with Stochastic Light Curves}

We have used two methods to assign stars to the stochastic light curve class, with each method having its own uncertainties. One method uses quantitative formulae to derive metrics for the light curves (Cody et al. 2014); however, the formulae are empirical and the boundaries are to some extent arbitrary. The other method is entirely qualitative and is based on "by eye" classification of the light curves by sorting them into groups which seem to have similar properties. The light curves shown here in Figure 20 match the algorithmic definition to be in the stochastic light curve class, but did not pass our "by eye" classification scheme. They therefore are not included in our final list of stars with stochastic light curves (Table 2). See discussion in Section 3.

\section{A.2. Spectral Feature Variability and Light Curve Morphology}

In Section 4.1, we showed that when we had appropriate synoptic high-resolution spectroscopy, the stars with stochastic light curves have veiling levels which vary with time and are quantitatively consistent with the observed photometric variability. We also showed that the Li I $6708 \AA$ doublet-the strongest absorption feature in our spectra-can be used as a veiling indicator (because its equivalent width variations correlate well with the equivalent width variations of other absorption features in our spectra) and that the variations in the lithium equivalent widths also correlate well with variations in the strength of the He I 6678 emission line.

In this section, we wish to show that these same spectral features can also be used as diagnostics of the physical processes underlying the variability for other light curve classes. For this purpose, we have selected six stars with disparate light curve morphologies and for which (a) the 

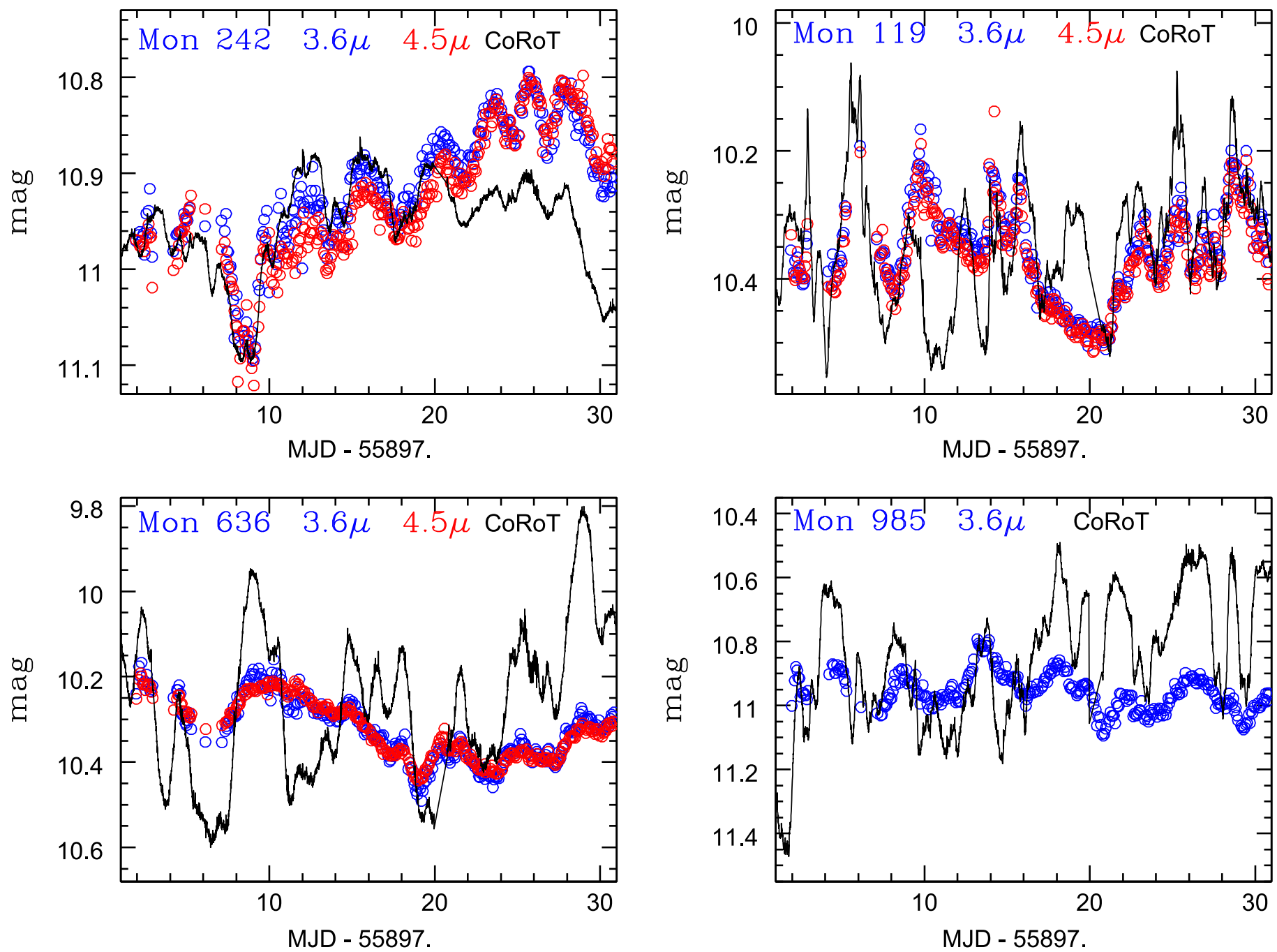

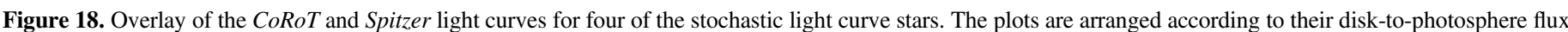

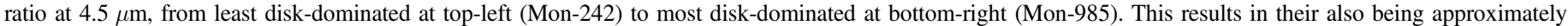

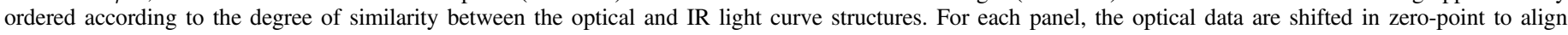
vertically with the IR data-the optical and IR delta-mag for full scale is the same.

CoRoT light curve amplitude in 2011 is at least $0.10 \mathrm{mag}$ (in order to be comparable to the typical amplitudes for the stochastic stars and in order to ensure a strong signal in our spectral indices if variable accretion drives the light curve morphology) and (b) we have multi-epoch VLT/FLAMES spectra in 2011. These six stars include (1) a WTTS with a periodic, stable light curve morphology which we attribute to cold spots; (b) a CTTS having a periodic light curve which we would also normally attribute to cold spots; (c) two CTTS whose CoRoT light curves show quasi-periodic structures which we attribute to hot spots; and (d) two CTTS whose CoRoT light curves have unusual morphologies which do not obviously align well with our standard light curve classes. Figures 21 and 22 show the CoRoT light curves, the correlation between lithium equivalent width and $\mathrm{He}_{\text {I }} 6678 \AA$, and the correlation between lithium equivalent width and the equivalent width of other relatively strong absorption features in our echelle order.

For these plots, as well as all the other similar plots in this paper, we have utilized sky-subtracted spectra. That is, for each object fiber, we have found the nearest sky fiber (which could be up to a few arcminutes away) and subtracted the latter from the former. Due to the spatially varying nebulosity, this is a necessarily imperfect process. For the absorption line and $\mathrm{He}_{\mathrm{I}}$ emission line measures of most interest here, sky subtraction primarily is needed in order to minimize any contribution from scattered moon light. The 2013 spectra were taken almost entirely during a dark run, and therefore the sky fiber continuum levels are very low and the uncertainty in sky correction has very little possible impact. For 2011, however, spectra were taken at all lunar phases, and the sky continuum level varies significantly and so could impact equivalent widths for faint stars. This is probably the largest source of uncertainty in our plots, though we believe it should not impact any of our conclusions. For the $\mathrm{He}_{\mathrm{I}} 6678 \AA$ A equivalent width, an additional source of uncertainty is that there is an $\mathrm{Fe}_{\mathrm{I}}$ absorption feature almost coincident in wavelength with the $\mathrm{He}$ I line, and whose strength is not negligible with respect to the $\mathrm{He}$ I line we normally see. We have corrected our measured $\mathrm{He}$ I equivalent widths for this $\mathrm{Fe}_{\mathrm{I}}$ feature by measuring the strength of the latter line in WTTS as a function of spectral type, and then subtracting this amount from the measured $\mathrm{He}$ I equivalent 

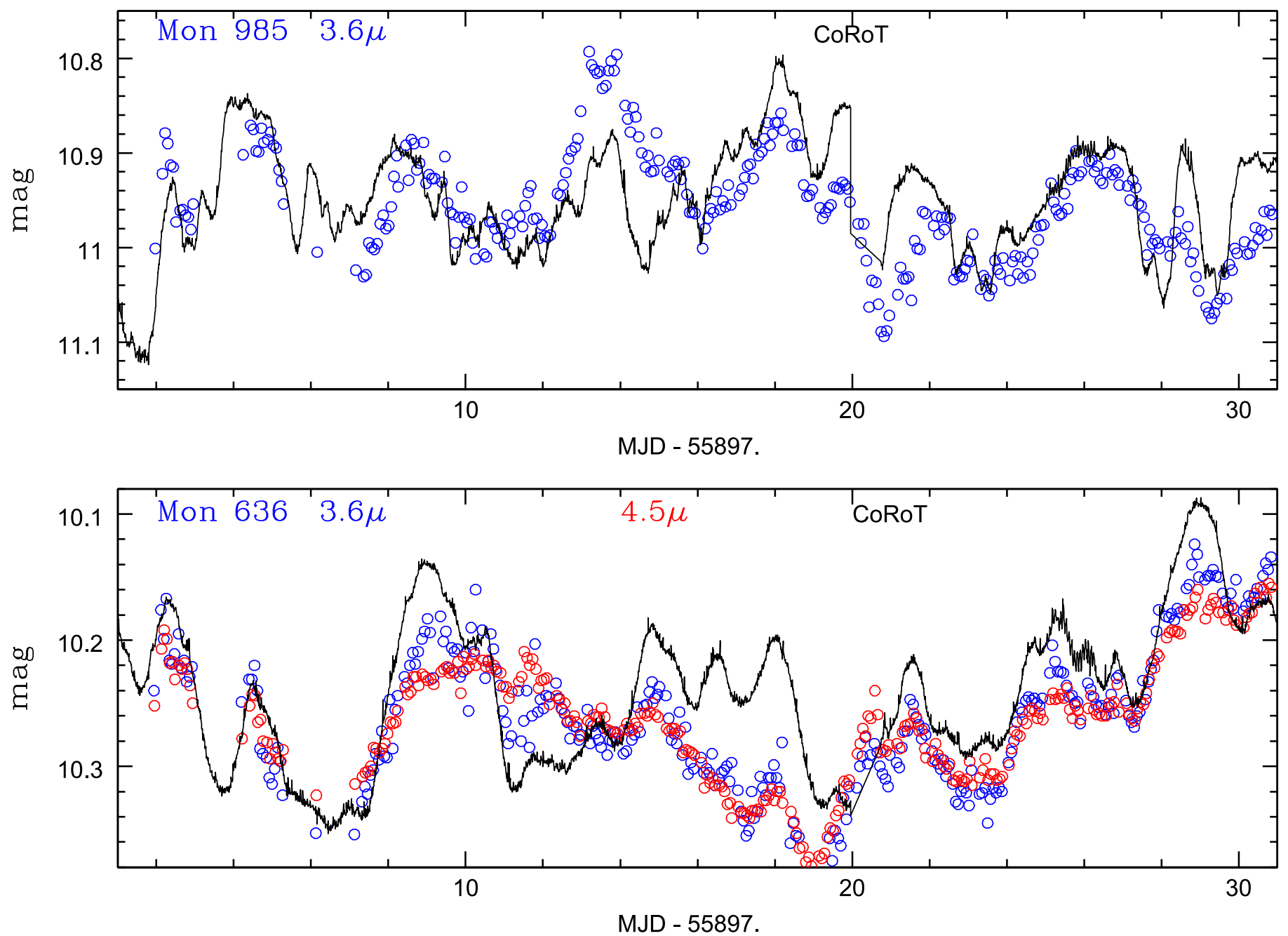

Figure 19. Overlay of the CoRoT and Spitzer light curves for Mon-636 and Mon-985, where in this case the $y$-axis full-scale for the IRAC data is one third that for the CoRoT data (e.g., for Mon-985, full scale for the IRAC data is 0.4 mag, whereas it is 1.2 mag for the CoRoT data). A slowly varying baseline has been subtracted from the Mon-636 CoRoT data in order to better visualize the short-timescale correlation of the optical and IR light curves.

widths for the stochastic stars. The Fe I equivalent widths range from $0.15 \AA$ at $\mathrm{G} 2$, increasing to about $0.26 \AA$ at $\mathrm{M} 0$, and then decreasing to $0.12 \AA$ at $\mathrm{M} 2$.

The plots for Mon-1274 (WTTS) and Mon-103 (CTTS) show these stars have little or no veiling variability despite CoRoT light curve amplitudes of $\sim 0.2$ mag. The rightmost plot for each star shows that He I 6678 is not in emission. The similar properties for the two stars indicates that cold spots can dominate the light curve morphology of CTTS, as has been advocated previously (e.g., Herbst et al. 1994). The dispersion in the plotted points for the center and right-hand side (rhs) plots for these two stars is primarily due to measurement uncertainties.

The CoRoT light curves of Mon-168 and Mon-370 (both CTTS) were selected on morphological grounds as candidates for rotational modulation of hot spots whose size/temperature vary significantly over the CoRoT campaign duration. This results in semi-sinusoidal waveforms that are more structured than in the case of cold-spots and also are less stable in time. The spectral indices plots confirm that these stars have variable veiling and $\mathrm{He} \mathrm{I}$ in emission, and that the absorption line veiling and He I emission are correlated. We therefore confirm that hot spots are the driver of the CoRoT light curve morphology, and the period derived for these stars is the photospheric rotational period.

Finally, Mon-498 and Mon-1199 (also both CTTS) have CoRoT light curves whose morphology are unusual and not completely consistent with any of our light curve classes. The center plot for these two stars (lithium versus "other" absorption line equivalent widths) shows no significant correlated variability, and hence no firm evidence for accretion variability. The rhs plots do show a possible correlation between the lithium equivalent width and $\mathrm{He}_{\mathrm{I}} 6678 \AA$. However, the inferred extra continuum flux would only be $\sim 5 \%-10 \%$ of the stellar photosphere, thus only about half of the CoRoT amplitude for Mon-498 and only a very small fraction of the CoRoT amplitude for Mon-1199 could be attributed to accretion variability. Instead, we suspect that cold spots plus one or more short-duration flux dips due to variable extinction (at day 5906 and possibly day 5930) best explain the light curve for Mon-498. The lack of veiling variability and the quite large amplitude of the photometric variations for Mon1199 argue that its light curve variations are primarily due to variable extinction arising from MRI or other instabilities in its inner circumstellar disk temporarily lofting more dust into the disk photosphere. 

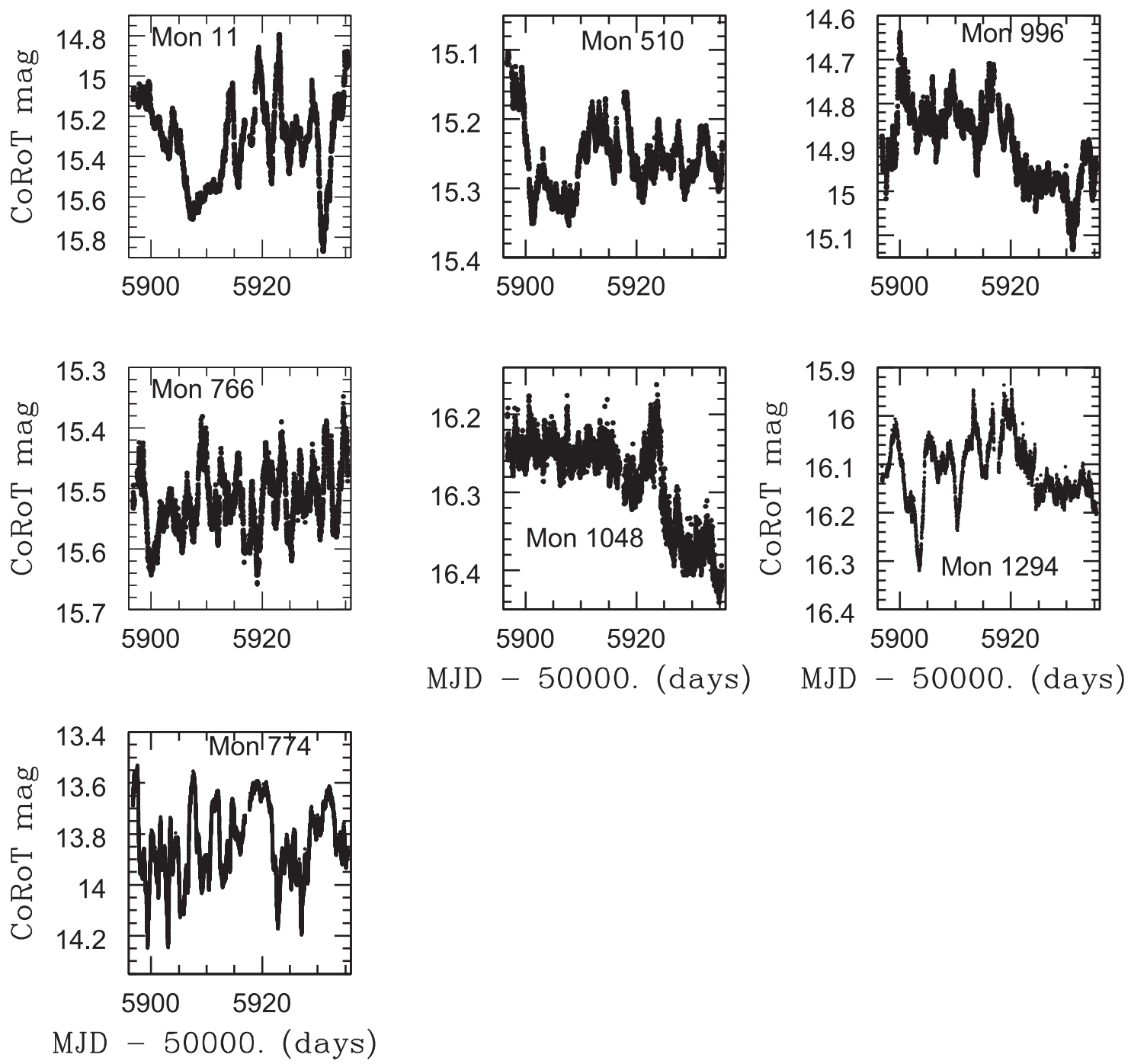

Figure 20. CoRoT data for stars with $Q>0.6$ and $-0.25<M<0.25$ (see Figure 4) that we believe do not belong in the stochastic light curve class. We instead classify Mon-11, Mon-510, Mon-996, Mon-766, and Mon-1048 as accretion-burst dominated; Mon-1294 as having light curves exhibiting both accretion bursts and flux dips due to variable extinction; and the 2011 light curve for Mon-774 as probably due to aperiodic extinction variations (see Section 3).

Stars whose light curves are dominated by deep flux dips produced by variable extinction events form another common CTTS light curve class. These flux dips can either be periodic (like AA Tau), and likely due to our line of sight being occulted by a warp in the inner circumstellar disk, or aperiodic, and possibly due to instabilities temporarily lifting more dust into the upper layers of the inner disk. Many stars appear to cycle back and forth between these two light curve morphologies on long (year or years) timescales (McGinnis et al. 2015). Based on their UV excesses, mean veiling and $\mathrm{H} \alpha$ equivalent widths, these stars have similar mean mass accretion rates as the stochastic light curve class members, and therefore we might expect similar veiling variability. We have investigated this by measuring the absorption line equivalent widths for eight of the variable extinction stars; Figure 23 shows plots of the correlation between $\mathrm{Li}$ I 6708 equivalent width and the other absorption lines (plotted in the same way as was done for the stochastic stars in Figure 8) for four of these stars. In summary, we find that the AA Tau and aperiodic flux dip stars show a range of accretion variability. The data for the two AA Tau stars with the largest measured veiling variability (Mon-811 and Mon-1037) are shown in the top of Figure 23. At the bottom of Figure 23, we show plots for two of the McGinnis et al. (2015) stars that show essentially undetectable veiling variability $(<5 \%$ variation in the lithium equivalent width). Most of the variable extinction stars in fact show undetected or barely detected $(<10 \%)$ variations in their veiling levels. This emphasizes that their large light curve amplitudes (often more than $0.5 \mathrm{mag}$, peak to peak) must be driven by some process other than variable accretion.

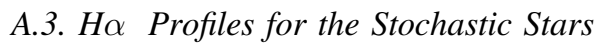

Our VLT/FLAMES spectra only have a wavelength coverage of about $350 \AA$. In the echelle order, we have only two lines normally in emission for CTTS are present- $\mathrm{H} \alpha$ and $\mathrm{He}_{\mathrm{I}}$ $6678 \AA$. For eight of the stochastic stars, we have multi-epoch data with either 12 or 20 spectra, depending on the year. In Figure 12, we overplot the entire set of profiles for two of the stars with multi-epoch data. Here (Figure 24), we provide single epoch $\mathrm{H} \alpha$ profiles for "representative" profiles for each of the stars for which we have VLT/FLAMES spectra. Where 

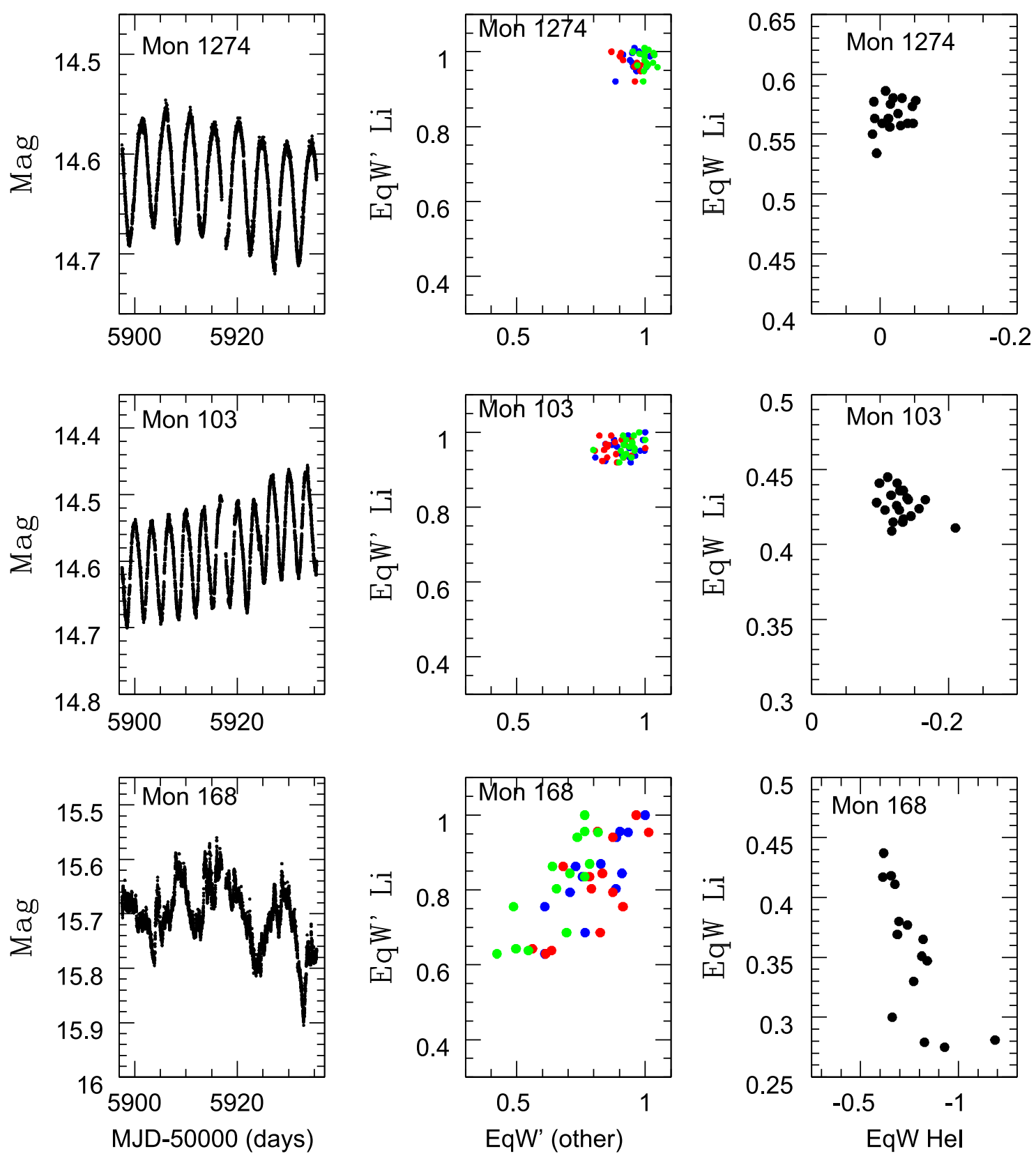

Figure 21. CoRoT light curves and spectral indices plots for three NGC 2264 members: (a) Mon-1274, a WTTS with a typical cold-spot light curve; (b) Mon-103, a CTTS with a cold-spot light curve; and (c) Mon-168, a CTTS with a hot-spot light curve (see text). In the middle plot in each row, the different colors correspond to different absorption features; also, the individual equivalent widths have been divided by the maximum equivalent width measured for that feature, in order to allow multiple features to be over-plotted.

we have multi-epoch data, we have tried to choose an epoch which shows features which are present in the majority of the epochs.

\section{A.4. Spectral Types Used in Figure 13}

In Table 5 we provide the spectral types used to construct the spectral type histograms for the accretion burst, variable extinction and short-duration flux dips classes shown in Figure 13. The spectral types for the stochastic class are provided in Table 2. For all four groups, where possible we use published spectral types, as described in Cody et al.
(2014). For a few of the short-duration flux dip stars, we have derived spectral type estimates from our own spectra (Stauffer et al. 2015). Where actual spectral types were not available, we have estimated spectral type by adopting the estimates for stellar luminosity and radius described in Venuti et al. (2014); combining those two quantities and the Stefan-Boltzmann law to estimate the effective temperature of the star; and then using the spectral type to $T_{\text {eff }}$ conversion for young stars provided in Pecaut \& Mamajek (2013). We also provide $\mathrm{H} \alpha$ equivalent widths for the variable extinction and accretion burst members in the table, because those numbers are used in Section 3. The $\mathrm{H} \alpha$ data are generally 
Table 5

Spectral Type Estimates for Additional Light Curve Classes

\begin{tabular}{|c|c|c|c|c|c|c|c|}
\hline Mon-ID ${ }^{\mathrm{a}}$ & Spectral Type $^{\mathrm{d}}$ & $\mathrm{H} \alpha \mathrm{EW}^{\mathrm{e}}$ & Mon-ID ${ }^{b}$ & Spectral Type ${ }^{\mathrm{d}}$ & $\mathrm{H} \alpha \mathrm{EW}^{\mathrm{e}}$ & Mon-ID ${ }^{c}$ & Spectral Type $^{\mathrm{d}}$ \\
\hline CSIMon-000007 & K7 & -12 & CSIMon-000126 & M0 & -26 & CSIMon-000021 & K5 \\
\hline CSIMon-000011 & K7 & -58 & CSIMon-000250 & K3 & -15 & CSIMon-000056 & K5 \\
\hline CSIMon-000117 & $\mathrm{M} 2.5$ & -353 & CSIMon-000296 & $\mathrm{K} 2$ & -11 & CSIMon-000314 & M3 \\
\hline CSIMon-000185 & $\mathrm{K} 4$ & -59 & CSIMon-000297 & $\mathrm{K} 2$ & -7 & CSIMon-000378 & K5.5 \\
\hline CSIMon-000260 & K7 & -62 & CSIMon-000325 & G6 & +0.2 & CSIMon-000566 & M3.5 \\
\hline CSIMon-000341 & M0.5 & -161 & CSIMon-000358 & M3* & -6 & CSIMon-0001076 & M1 \\
\hline CSIMon-000406 & $\mathrm{K} 3^{*}$ & -46 & CSIMon-000379 & $\mathrm{K} 2$ & -36 & CSIMon-001131 & M1.5+ \\
\hline CSIMon-000412 & M1 & -31 & CSIMon-000433 & $\mathrm{M}^{*}$ & -7 & CSIMon-001165 & M1.5+ \\
\hline CSIMon-000469 & K7 & -237 & CSIMon-000441 & M2* & -34 & CSIMon-001580 & $\mathrm{M}^{*}$ \\
\hline CSIMon-000474 & G5 & -105 & CSIMon-000456 & K4 & -13 & CSIMon-006975 & $\mathrm{M} 2.5+$ \\
\hline CSIMon-000510 & M0 & -102 & CSIMon-000650 & K1 & -6 & $\cdots$ & $\ldots$ \\
\hline CSIMon-000567 & K3 & -84 & CSIMon-000660 & K4 & -17 & CSIMon-000119 & K6 \\
\hline CSIMon-000808 & K4 & -50 & CSIMon-000667 & $\mathrm{K} 3^{*}$ & -0.1 & CSIMon-000342 & M4 \\
\hline CSIMon-000860 & M2.5 & -261 & CSIMon-000676 & $\mathrm{K} 4^{*}$ & -1 & CSIMon-000577 & K1 \\
\hline CSIMon-000877 & K4 & -91 & CSIMon-000681 & $\mathrm{K} 3^{*}$ & -26 & CSIMon-001038 & M0 \\
\hline CSIMon-000919 & M4 & -80 & CSIMon-000717 & M0.5 & -24 & CSIMon-001114 & M1.5 \\
\hline CSIMon-000945 & $\mathrm{K} 4$ & -66 & CSIMon-000774 & $\mathrm{K} 2.5$ & -14 & $\cdots$ & $\cdots$ \\
\hline CSIMon-000996 & K7 & -25 & CSIMon-000811 & K6 & -27 & $\cdots$ & $\cdots$ \\
\hline CSIMon-001022 & K4 & -46 & CSIMon-000824 & K4 & -2 & $\ldots$ & $\cdots$ \\
\hline CSIMon-001174 & M2 & -130 & CSIMon-000928 & M0 & -16 & $\ldots$ & $\cdots$ \\
\hline CSIMon-001187 & M2* & -8 & CSIMon-001037 & K1 & -22 & $\cdots$ & $\cdots$ \\
\hline CSIMon-001217 & K4 & -87 & CSIMon-001140 & $\mathrm{K} 3^{*}$ & -32 & $\cdots$ & $\cdots$ \\
\hline CSIMon-001573 & $\mathrm{K} 9^{*}$ & -26 & CSIMon-001144 & K5 & -50 & $\ldots$ & $\ldots$ \\
\hline CSIMon-000766 & M0.5 & -70 & CSIMon-001296 & $\mathrm{K} 6^{*}$ & -16 & $\ldots$ & $\ldots$ \\
\hline CSIMon-001048 & M3* & $\ldots$ & CSIMon-001308 & $\mathrm{K} 8^{*}$ & -41 & $\ldots$ & $\cdots$ \\
\hline
\end{tabular}

Notes.

a These stars all have accretion-burst dominated optical light curves (Stauffer et al. 2014).

b These stars all have variable extinction dominated optical light curves (McGinnis et al. 2015).

${ }^{c}$ These stars all have optical light curves containing short-duration, shallow flux dips (Stauffer et al. 2015).

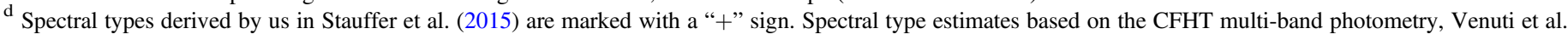
(2014) and Pecaut \& Mamajek (2013) are marked with a “*” sign. Other spectral types from Dahm \& Simon (2005) or Makidon et al. (2004).

${ }^{\mathrm{e}} \mathrm{H} \alpha$ equivalent widths primarily from Dahm \& Simon (2005), otherwise from our own spectra.

from the literature (Cody et al. 2014); when no literature value was available, we have measured representative $\mathrm{H} \alpha$ equivalent widths from our own VLT spectra, from the ESOGaia spectra, or from MMT Hectochelle spectra described in Furesz et al. (2006).

\section{A.5. Derivation of Projected Rotational Velocities $\left(v \sin i^{\prime} s\right)$ from the VLT Spectra}

We have derived $v \sin i$ estimates in two ways for the CTTS in NGC 2264 for which we have VLT/FLAMES spectra. Our initial $v \sin i$ estimation begins by co-adding all epochs of spectra for each star to produce a high $\mathrm{S} / \mathrm{N}$ mean spectrum. For the adopted spectral type for each star, we produce a synthetic spectrum at the FLAMES spectral resolution, and then convolve that spectrum with a rotational broadening function, using software routines available in SME (Valenti \& Piskunov 1996), Synth3 and Binmag3 (Kochukhov 2007). We do this for a range in adopted rotational velocities, and compare the model spectra to the observed spectrum in a least-squares sense. The $v \sin i$ we adopt is that for the model whose spectrum matches most closely the observed spectrum. The formal uncertainties in the derived $v \sin i$ 's are generally $2-3 \mathrm{~km} \mathrm{~s}^{-1}$. We note that the model spectra assume solar abundance, hence essentially no feature is present for the lithium $6708 \AA$ doublet; these $v \sin i$ 's therefore are based on all the other absorption features in the FLAMES $\mathrm{H} \alpha$ order.
After deriving $v \sin i$ 's in this manner for all of our NGC 2264 CTTS, we plotted those $v \sin i$ 's versus the mean FWHM of the lithium $6708 \AA$ doublet for each star (i.e., we measured the FWHM at each epoch, and then took the average of those values; the FWHM for the stochastic stars ranges from 0.7 to $1.8 \AA$, with rms for the dozen or more epochs for a given star of order $0.03 \AA$ ). The plot shows the expected relationship, but with a larger than anticipated scatter for stars with small $v \sin i$. As one approaches the resolution limit of the spectrograph, one of course expects an increase in scatter, but it was also possible that by averaging spectra for the $v \sin i$ analysis we could artifically broaden lines (e.g., for an SB1). Therefore, we adopted a second procedure to estimate $v \sin i$ based on combining the lithium FWHM data and the original $v \sin i$ data to provide a calibration. For this purpose, we fit a calibration curve to the plot of $v \sin i$ versus FWHM. Then, for each star, we used that calibration curve and the measured mean lithium FWHM to provide a new $v \sin i$ estimate. Because the lithium $6708 \AA$ line is always the strongest absorption feature in these spectra, for faint and/or slowly rotating stars, this latter $v \sin i$ estimate could be more reliable than the initial estimate. Table 4 provides both $v \sin i$ estimates-column 7 provides the initial $v \sin i$ estimate, and column 8 the $v \sin i$ estimate derived from the measured lithium FWHM.

Comparison of the two $v \sin i$ estimates in Table 4 shows generally good correspondence. However, a few stars show 

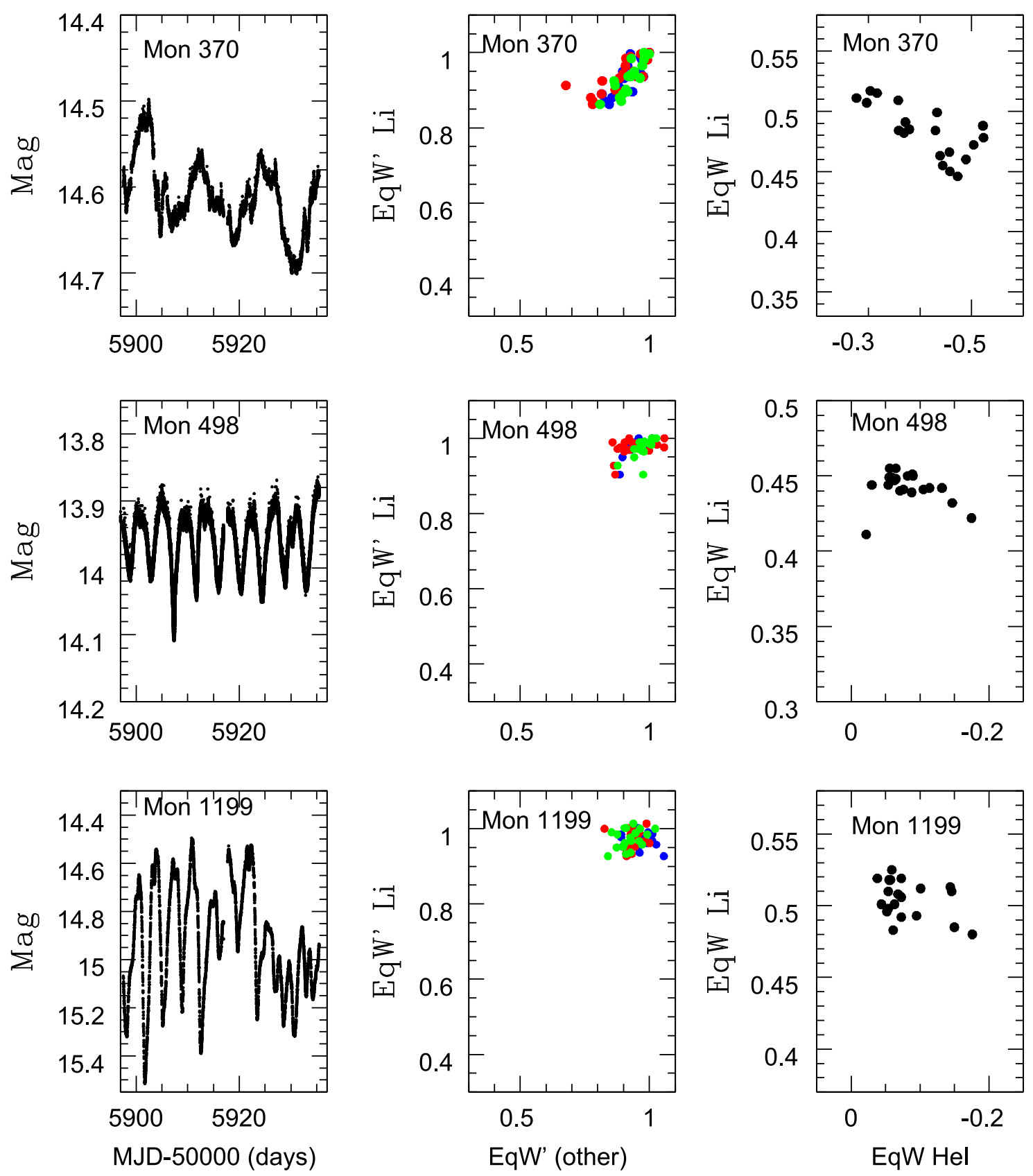

Figure 22. CoRoT light curves and spectral indices plots for three additional NGC 2264 members: (a) Mon-370, another CTTS with a hot-spot light curve; (b) Mon498 and (c) Mon-1199- two CTTS whose light curves are unusual and where we are using these diagrams to help determine the primary physical mechanism driving their optical variability. See Figures 8 and 21 captions for further details.

significant differences, with Mon-1234 having the most significant discrepancy ( 24.0 versus $15.9 \mathrm{~km} \mathrm{~s}^{-1}$ ). The spectral synthesis method yields essentially the same $v \sin i$ for Mon-636 and Mon-1234, while the lithium FWHM method yields 24.9 and $15.9 \mathrm{~km} \mathrm{~s}^{-1}$, respectively. The mean and rms FWHM for the two stars are $0.827 \pm 0.036$ and $0.725 \pm 0.024$, respectively, with none of the Mon-1234 FWHM values being as large as the smallest FWHM measurement for Mon-636. The lithium widths preclude these stars from having the same $v \sin i$. Based on this and similar comparisons, we adopt the $v \sin i$ 's derived from the lithium width as our best estimates of $v \sin i$, and we use those estimates in the plots in the main body of the paper. 

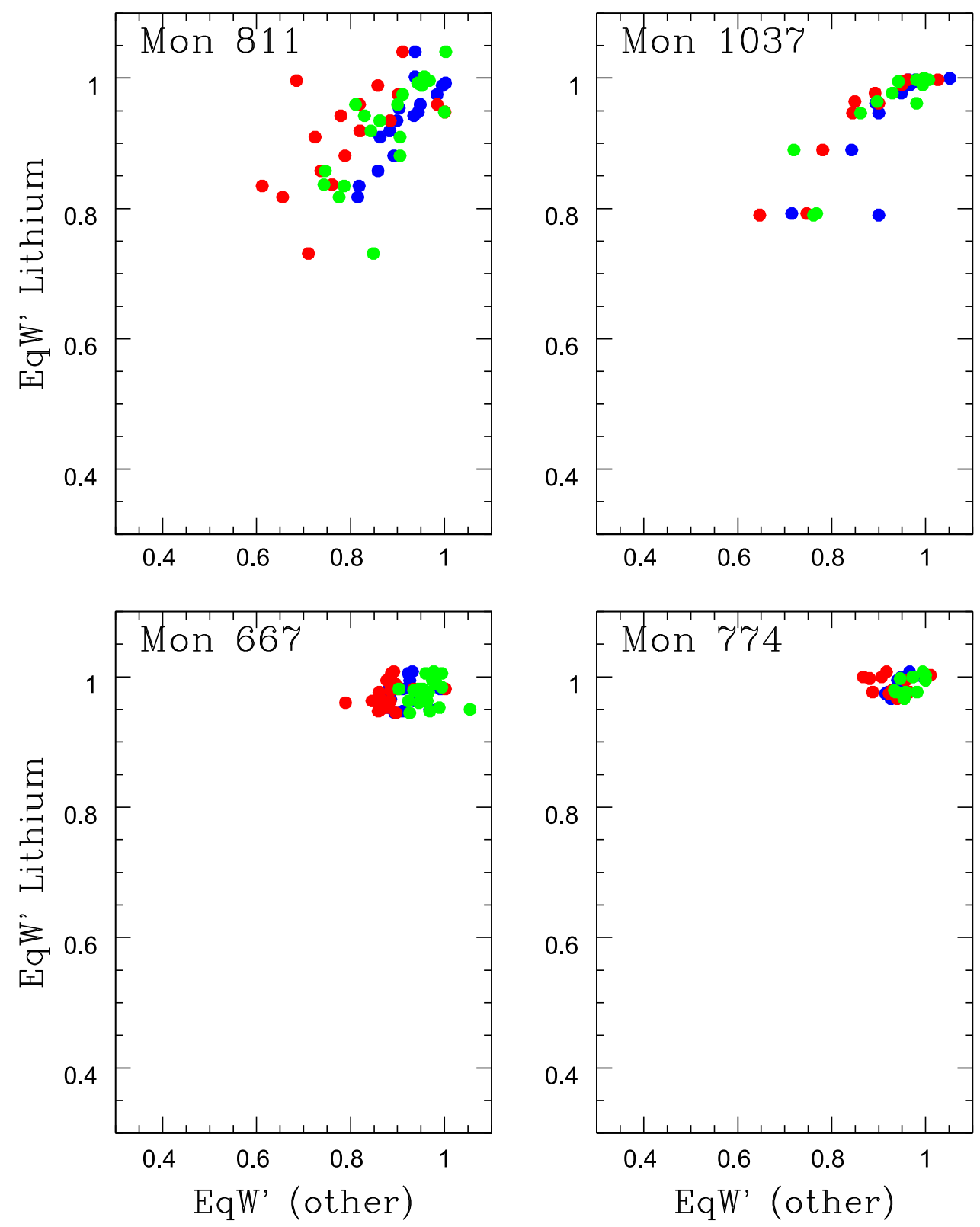

Figure 23. Correlation between the equivalent width for the Li I $6708 \AA$ doublet and other absorption lines present in the same FLAMES echelle order, for four CTTS whose CoRoT light curves are dominated by variable extinction events. The equivalent widths at each epoch have been divided by the maximum equivalent width for that feature, in order to allow data for several features to be over-plotted. The top two panels show stars with strong veiling variability; their He I 6678 emission line equivalent widths also vary and are well-correlated with the variations in the veiling levels from the absorption features. The bottom two plots show two stars with similar CoRoT light curve morphology, but where there is no detectable veiling variability. 

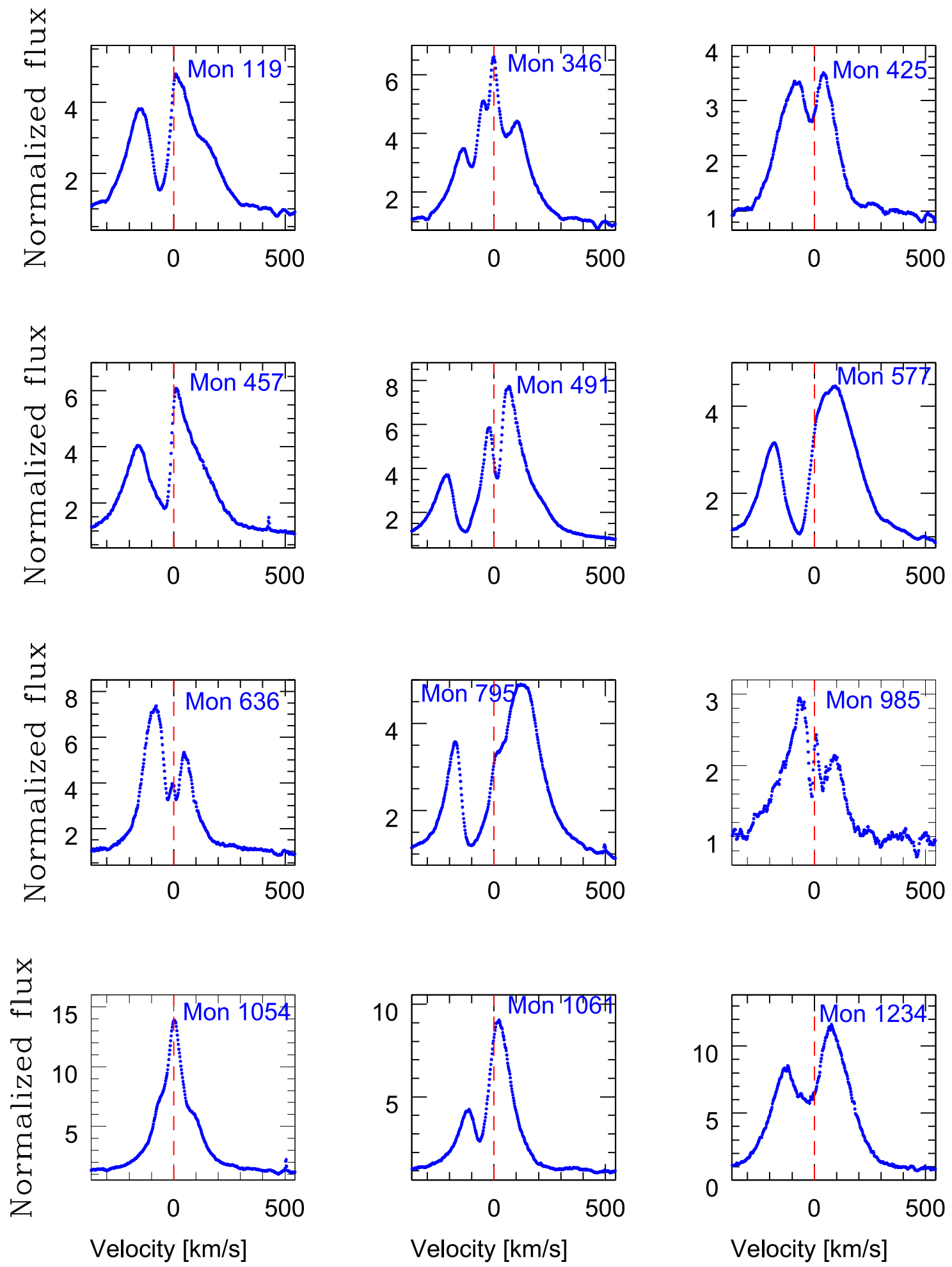

Figure 24. $\mathrm{H} \alpha$ profiles of the stars whose CoRoT light curves are categorized as stochastic, for those stars for which we have available high resolution spectra. The vertical dashed red line marks rest velocity for $\mathrm{H} \alpha$. We omit an $\mathrm{H} \alpha$ profile for Mon-613 here (to make the figure more compact)-our multi-epoch $\mathrm{H} \alpha$ profiles for this star are shown in Figure 12. 


\section{REFERENCES}

Alencar, S. H. P., Teixeria, P. S., Guimarães, M., et al. 2010, A\&A, 519, A88 Allen, L. E., Calvet, N., D’Alessio, P., et al. 2004, ApJS, 154, 363

Ambartsumian, V. 1954, MSRSL, 1, 293

Baraffe, I., \& Chabrier, G. 2010, A\&A, 521, 44

Barrado, D., Garcia-Lopez, R., Severino, G., \& Gomez, M. 2001, A\&A, 371,652

Basri, G., \& Batalha, N. 1990, ApJ, 363, 654

Basri, G., Martín, E., \& Bertout, C. 1991, A\&A, 252, 625

Beristain, G., Edwards, S., \& Kwan, J. 2001, ApJ, 551, 1037

Bertout, C. 2000, A\&A, 363, 984

Blinova, A., Romanova, M., \& Lovelace, R. 2015, arXiv:1501.01948

Bouvier, J., Chelli, A., Allain, S., et al. 1999, A\&A, 349, 619

Bouvier, J., Grankin, K., Alencar, S., et al. 2003, A\&A, 409, 169

Bouvier, J., Grankin, K., Ellerbroek, L., et al. 2013, A\&A, 557, A77

Carpenter, J., Hillenbrand, L., \& Skrutskie, M. 2001, AJ, 121, 3160

Cody, A., Stauffer, J., Baglin, A., et al. 2014, AJ, 147, 82

Dahm, S., \& Simon, T. 2005, AJ, 129, 829

Donati, J.-F., Skelly, M., Bouvier, J., et al. 2010, MNRAS, 409, 1347

Findeisen, K., Cody, A., \& Hillenbrand, L. 2015, ApJ, 798, 89

Furesz, G., Hartmann, L., Szentgyorgyi, A., et al. 2006, ApJ, 648, 1090

Ghosh, P., \& Lamb, F. 1978, ApJL, 223, L83

Gregory, S., Donati, J.-F., Morin, J., et al. 2012, ApJ, 755, 97

Haro, G., \& Herbig, G. 1955, BOTT, 21, 33

Hartigan, P., Hartmann, L., Kenyon, S., et al. 1989, ApJS, 70, 899

Herbst, W., Booth, J., Koret, D., et al. 1987, AJ, 94, 137

Herbst, W., Herbst, D., Grossman, E., \& Weinstein, D. 1994, AJ, 108, 1906

Herbst, W., Holtzman, J., \& Phelps, B. 1982, AJ, 87, 1710

Herbst, W., Rhode, K., Hillenbrand, L., \& Curran, G. 2000, AJ, 119, 261

Holtzman, J., Herbst, W., \& Booth, J. 1986, AJ, 92, 138

Johns-Krull, C., Chen, W., Valenti, J., et al. 2013, ApJ, 765, 11

Joy, A. 1945, ApJ, 102, 168

Kochukhov, O. 2007, in Physics of Magnetic Stars, ed. I. I. Romanyuk, \& D. O. Kudryavtsev, 109

Königl, A. 1991, ApJL, 370, L39
Kraft, R. 1970, in Spectroscopic Astrophysics: An Assessment of the Contributions of Otto Struve, ed. G. H. Herbig (Berkeley: Univ. California Press), 385

Kurosawa, R., Harries, T., \& Symington, N. 2006, MNRAS, 370, 580

Kurosawa, R., \& Romanova, M. 2013, MNRAS, 431, 2673

Lamm, M., Mundt, R., Bailer-Jones, C., \& Herbst, W. 2005, A\&A, 430, 1005

Lima, G., Alencar, S., Calvet, N., et al. 2010, A\&A, 522, 104

Makidon, R., Rebull, L., Strom, S., et al. 2004, AJ, 127, 2228

McGinnis, P., Alencar, S., Guimaraes, M., et al. 2015, A\&A, 577, 11

Moraux, E., Artemenko, S., Bouvier, J., et al. 2013, A\&A, 560, 13

Pecaut, M., \& Mamajek, E. 2013, ApJS, 208, 9

Randich, S., Gilmore, G., \& Gaia-ESO, C. 2013, Msngr, 154, 47

Rebull, L. 2001, AJ, 121, 1676

Rebull, L., Cody, A., Covey, K., et al. 2014, AJ, 148, 92

Rydgren, E., \& Vrba, F. 1983, ApJ, 267, 191

Salpeter, E. 1954, in Les Processus Nucleaires dan les Astres, Communications

Presentees au cinquieme Colloque International d'Astrophysique tenu a Liege, 113

Schmidt, J. 1861, AN, 55, 91

Schmidt, J. 1866, AN, 68, 69

Sousa, A., Alencar, S., Bouvier, J., et al. 2015, arXiv:1509.05354

Stauffer, J., Cody, A., Baglin, A., et al. 2014, AJ, 147, 83

Stauffer, J., Cody, A., McGinnis, P., et al. 2015, AJ, 149, 130

Stetson, P. 1996, PASP, 108, 851

Sung, H., Bessell, M., Chun, M., et al. 2008, AJ, 135, 441

Sung, H., Stauffer, J., \& Bessell, M. 2009, AJ, 138, 1116

Teixeira, P., Lada, C., Marengo, M., \& Lada, E. 2012, A\&A, 540, 83

Terquem, C., \& Papaloizou, J. 2000, A\&A, 360, 1031

Valenti, J., \& Piskunov, N. 1996, A\&AS, 118, 595

Venuti, L., Bouvier, J., Flaccomio, E., et al. 2014, A\&A, 570, 82

Venuti, L., Bouvier, J., Irwin, J., et al. 2015, A\&A, 581, 66

Vrba, F., Rydgren, A., Chugainov, P., et al. 1986, ApJ, 306, 199

Vrba, F., Rydgren, A., Chugainov, P., et al. 1989, AJ, 97, 483

Walker, M. 1956, ApJS, 2, 365

Walker, M. 1972, ApJ, 175, 89

Whitney, B., Robitaille, J., Bjorkman, J., et al. 2013, ApJS, 207, 30

Wolf, B., Appenzeller, I., \& Bertout, C. 1977, A\&A, 58, 163 